\title{
MicroRNA-654-5p suppresses ovarian cancer development impacting on MYC, WNT and AKT pathways
}

\author{
Blanca Majem ${ }^{1,18}$ - Alfonso Parrilla ${ }^{1}$ Carlos Jiménez ${ }^{2}$ - Leticia Suárez-Cabrera ${ }^{1}$ - Marta Barber ${ }^{1}$ Andrea Marín ${ }^{1}$. \\ Josep Castellví ${ }^{3}$ - Gabriel Tamayo ${ }^{1}$ - Gema Moreno-Bueno ${ }^{4,5}$ - Jordi Ponce ${ }^{6}$ - Xavier Matias-Guiu', ${ }^{7,8}$. \\ Francesc Alameda ${ }^{9} \cdot$ Ignacio Romero $^{10} \cdot$ José Luis Sánchez $^{11,12} \cdot$ Asunción Pérez-Benavente $^{11,12}$. \\ Sebastián Moran $\mathbb{1}^{13}$. Manel Esteller ${ }^{13,14,15}$ - Jaume Reventós ${ }^{8,16}$. Marina Rigau ${ }^{17}$. Antonio Gil-Moreno $\mathbb{D}^{11,12}$. \\ Miguel F. Segura $\mathbb{D}^{2} \cdot$ Anna Santamaría ${ }^{1}$
}

Received: 28 August 2018 / Revised: 28 January 2019 / Accepted: 16 April 2019

(c) The Author(s), under exclusive licence to Springer Nature Limited 2019

\begin{abstract}
Ovarian cancer is the most lethal gynecological malignancy due to the silent nature on its early onset and the rapid acquisition of drug resistance. Histologically heterogeneous, it includes several subtypes with different mutational landscapes, hampering the development of effective targeted therapies. Non-coding RNAs are emerging as potential new therapeutic targets in cancer. To search for a microRNA signature related to ovarian carcinomas and study its potential as effective targeted therapy, we examined the expression of 768 miRNA in a large collection of tumor samples and found miR-654-5p to be infraexpressed in ovarian serous carcinomas, the most common and aggressive type. Restoration of miR654-5p levels reduced tumor cell viability in vitro and in vivo and impaired sphere formation capacity and viability of ovarian cancer patient-derived ascitic cells ex vivo. CDCP1 and PLAGL2 oncogenes were found to be the most relevant direct miR-654-5p targets and both genes convey in a molecular signature associated with key cancer pathways relevant to ovarian tumorigenesis, such as MYC, WNT and AKT pathways. Together, we unveiled the tumor suppressor function of miR-654-5p, suggesting that its restoration or co-targeting of CDCP1 and PLAGL2 may be an effective therapeutic approach for ovarian cancer.
\end{abstract}

\section{Introduction}

Ovarian cancer (OC) is the most lethal gynecological malignancy [1] and includes different subtypes, being highgrade serous carcinoma (HGSC) the most common and aggressive subtype [2]. Despite the efforts devoted to improve early diagnosis [3, 4], the disease still remains largely incurable, in part due to the presence of ascites in advanced stages [5]. Over the past 50 years, the first-line chemotherapy for OC, composed mainly of platinum and taxane derivatives [6], has neither varied nor resulted into a significant improved overall survival [6]. Recently, targeted

Supplementary information The online version of this article (https:// doi.org/10.1038/s41388-019-0860-0) contains supplementary material, which is available to authorized users.

Anna Santamaría

anna.santamaria@vhir.org

Extended author information available on the last page of the article therapies against PI3K, MEK, EGFR and key regulators of the cell cycle, among others [7] are on the horizon of new treatments for OC. Still under the regimen of recurrent OC, some improvements have been achieved with the introduction in the therapeutic pipeline of anti-angiogenic agents [7], and PARP inhibitors for BRCA mutation carriers [8] and patients with a "BRCAness" phenotype [9]. However, and due to the heterogeneity of the disease, a deeper molecular understanding of the tumor biology is needed to generate novel and efficient therapies.

Recent evidence suggests that modulation of microRNA (miRNA) levels, a type of small ( 18-22 nt) non-coding RNAs, can be a novel therapeutic approach for cancer and other diseases [10] thanks to the ability of a particular miRNA to target multiple genes that might be altered in specific disease conditions. A considerable number of preclinical studies involving miRNA therapeutics have been conducted over the last years [11]. Several miRNA have been shown to be downregulated in ovarian tumors, associated to shorter or poorer overall survival and related to 
better response to chemotherapy [12-16]. However, only few of them have moved into clinical trials [10], such as the first phase I study in humans that has been completed in 2017 using miR-34a for the treatment of advanced solid tumors [17].

The heterogeneity of miRNA-detecting platforms and patient cohorts hamper the interpretation of the results across studies and the implementation of miRNAs as therapeutic tools in OC [12]. Here, we provide an extensive study of miRNA expression in more than 200 tissue samples, and the use of clinically representative models that better recapitulate the human disease. We have found that the restoration of miR-654 expression levels significantly inhibited cell proliferation and induced apoptosis in vitro, in vivo and in ex vivo patient derived-ascitic cell models. Further investigation into its mechanism of action showed that miR-654 altered the expression of CDCP1 and PLAGL2, thereby impacting on the oncogenic function of MYC, AKT and Wnt pathway, opening novel possibilities for the treatment of OC.

\section{Results}

\section{MmiR-654 is downregulated in ovarian tumors}

In an attempt to identify new targets for OC therapy, we analyzed miRNA expression levels in advanced-stage OC patients, under the arguably hypothesis that deregulated miRNA in shorter living patients could potentially be involved in the disease progression and serve as novel therapeutic targets. Patients were classified into two groups $(n=107)$ according to an overall survival of less than 3 or more than 8 years (short- and long-term survival; SSV and LSV, respectively; Supplementary Table 1a). We initially used a cohort of 27 OC FFPE primary tumors (Supplementary Fig. 1a) and profiled miRNA levels. Thirty-two miRNA were found differentially expressed between both groups (Fig. 1a, Supplementary Data S1). Unsupervised hierarchical clustering analysis showed that the majority of patients clustered according to their survival group (Fig. 1a). Next, we used an independent multicenter cohort of 80 FFPE samples (Supplementary Fig. 1a) to validate the identified miRNA (Fig. 1a, Supplementary Data S1), and found 4 miRNA, namely miR-654, miR-554, miR-409-3p and miR-127-3p that displayed higher levels in tumors from SSV compared to LSV patients with $p$-value $<0.05$ and fold change $>1.5$ (Supplementary Fig. 1b, c).

We then modulated the expression of the 4-validated miRNA in multiple OC cell lines and found that miR-654 overexpression decreased cell proliferation in all lines tested (Fig. 1b), while its inhibition increased proliferation in 3 of the 4 OC cell lines (Supplementary Fig. 1d). Despite this was in apparent contradiction with the observation that miR-654 levels were higher in SSV patients, the consequences of its modulation in vitro suggests that miR-654 presumably acts as a tumor suppressor in OC. Supporting this hypothesis we found lower levels of miR-654 in tumors compared to benign ovarian tissues (Fig. 1c) $(n=84$, Supplementary Table 1b), which were further decreased in the HGSC histology, the most aggressive type of OC (Fig. 1d). A small cohort from the initial screening, with extended clinical follow up, revealed that the difference between SSV $v s$. LSV samples, albeit significant in agreement to our initial observation, was within the lower expression range that tumoral samples display for miR-654 compared to benign samples (Fig. 1c). Kaplan-Meier survival curves from HGSC tumors (Supplementary Fig. 1c) reflected that high miR-654 expression related to poorer survival (Supplementary Fig. 1e), suggesting a prognostic role for miR654 levels within tumors. In addition, no differences were found when tumors where grouped as early- or advancedstage OC (Supplementary Fig. 1f), or between paired tumor and metastases samples from HGSC patients (Supplementary Fig. 1g) (Supplementary Table 1c), supporting the hypothesis that the reduction on miR-654 levels may be more related to the initiation of the disease, and points towards a tumor suppressor role for miR-654.

\section{MiR-654 promoter region is hypermethylated in OC cell lines and tissues}

MiR-654 is located in Chromosome 14, within the q32.31 chromosomal region, a region frequently altered in cancer [18-20], including OC [13]. Here, we found hypermethylation in the $\mathrm{CpG}$ islands of 79 and $89 \%$ of tumors and cell lines evaluated, respectively, with a $\beta$-value $\geq 0.5$ (Fig. 1e). Accordingly, the levels of miR-654 significantly raised upon DNA methyltransferase inhibition in OAW28 cells and the same tendency was observed for A2780 cells (Fig. 1f) (two of the cell lines that expressed low miR-654 levels, Supplementary Fig. 1h; and high levels of hypermethylation). This was accompanied by increased expression of MEG3, GBGT1 and MAGE-A3 positive control genes [21-23] (Supplementary Fig. 1i). Together, this suggests that hypermethylation could be responsible in part for the low miR-654 levels found in OC, despite other mechanisms may be regulating miR654 expression in OC.

\section{Overexpression of miR-654 reduces cell viability and sensitizes cells to paclitaxel in vitro}

The effects of miR-654 ectopic expression were analyzed and a $50 \%$ reduction in cell proliferation was observed in SKOV3 and A2780 cells in a miR-654 overexpression 

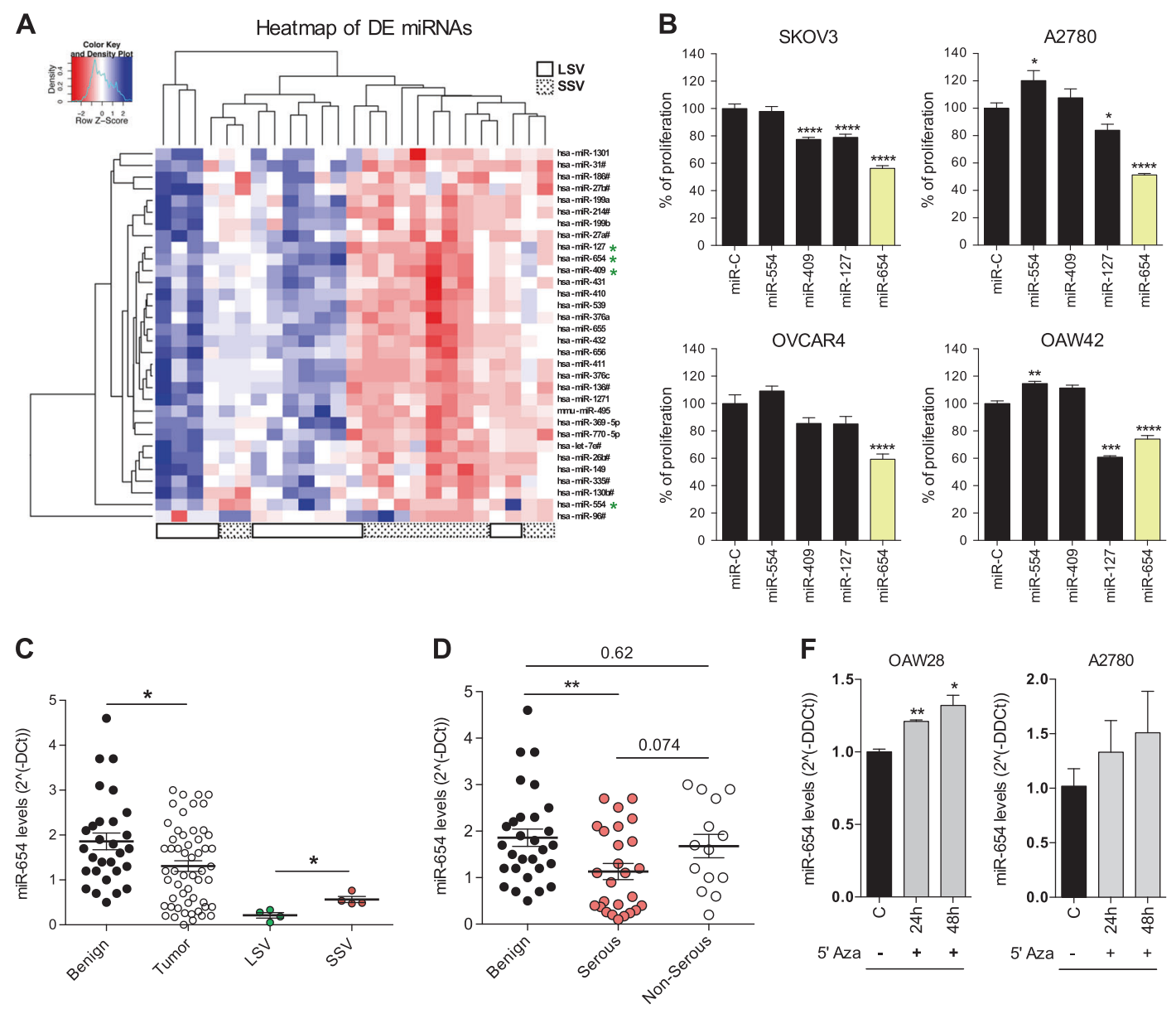

E
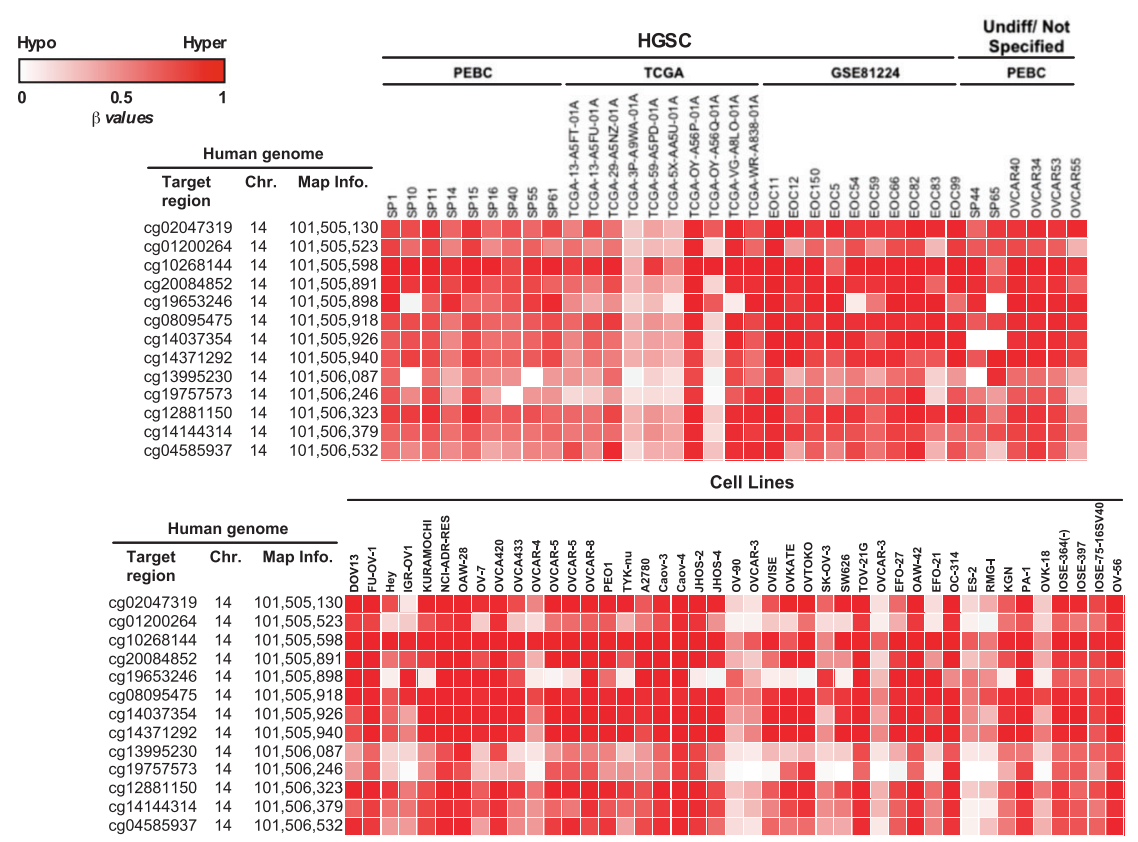

time-course (Fig. 2a, Supplementary Fig. 2a, b). Cell death was analyzed in 4 OC cell lines finding the number of apoptotic cells significantly increased upon miR-654 transfection compared to miR-Control-cells (Fig. 2b, c), which was confirmed by the cleavage of Caspase- 3 and its substrate PARP-1 (Fig. 2d). We found that miR-654 did not 
Fig. 1 Decreased miR-654 expression and hypermethylation of its locus are found in OC. a Unsupervised hierarchical clustering of the 32 differentially expressed miRNA $($ FDR $<0.05)$ found in the discovery phase ( $n=25$, as two patients were outliers; see also Supplementary Methods), in SSV compared to LSV patients. b Evaluation of the proliferation effect in miRNA mimic-transfected indicated OC cells $96 \mathrm{~h}$ post-transfection. Graph represents the mean \pm SEM of three independent experiments $(n=6 /$ condition $)$. c Relative miR-654 expression levels in benign lesions $(n=29)$ compared to ovarian tumors $(n=55)$; and in a cohort of LSV vs. SSV tumors $(n=8)$ randomly selected fro (a). d Relative miR-654 expression levels in ovarian tumors according to their histology. e Methylation analysis of the $13 \mathrm{CpG}$ sites of the miR-654 locus in OC cell lines (top) and tumors (bottom). f MiR-654 levels upon treatment with $1 \mu \mathrm{M}$ of 5AZA-2'-deoxycytidine at the indicated times. Graph represents the mean \pm SEM of 2 independent experiments. $* p<0.05 ; * * p<0.01$; $* * * p<0.001 ; * * * * p<0.0001 . p$ values were calculated using unpaired student's $t$-test for (b) and (f), and non-parametric MannWhitney tests for (c) and (d). See Supplementary Methods for statistics of (A)

induce cell death in the presence of the caspase-inhibitor $\mathrm{Q} /$ $\mathrm{VD} / \mathrm{OPH}$, thereby indicating that miR-654-induced cell death is caspase-dependent (Supplementary Fig. 2c). Furthermore, cell cycle profile analysis showed a subG1 peak at $96 \mathrm{~h}$ post-miR-654 transfection (Supplementary Fig. 2d), consistent with the increased cell death observed, and a slight increase in the percentage of cells in G1 (Supplementary Fig. 2e), suggesting that miR-654 overexpression caused an initial cell cycle delay that may add to the reduced proliferation observed. To test whether miR-654 sensitize cells to paclitaxel, SKOV3 and A2780 cells were treated with paclitaxel at different doses and transfected with miR-654, showing a synergistic effect in reducing cell viability (Fig. 2e, Supplementary Fig. 2f, g), and suggesting that the combination of miR-654 and paclitaxel may be a suitable therapy for OC.

\section{MiR-654 expression reduces the viability of $O C$ patient-derived ascitic cells}

Tumor ascites, which survive in anchorage-independent conditions growing as spheroids and drive OC progression at later stages [24], are a major source of disease recurrence. Patient-derived ascitic cells were grown ex vivo to evaluate the potential therapeutic role of miR-654 in advanced disease stages, finding that miR-654 overexpression significantly reduced the number, size and viability of spheroids (Fig. 2f-h), and increased spheroid caspase-dependent apoptosis (Fig. 2i). Altogether, this mirrored the previous results observed in OC cell lines and empowered in addition patient-derived ascites as ex vivo models to evaluate novel therapies for advanced-stage OC patients.

\section{MmiR-654 overexpression impairs tumor growth in vivo}

To recapitulate in vivo the results obtained in vitro, $2 \times 10^{6}$ miR-Control and miR-654 transfected SKOV3 cells were injected in the flank of nude mice ( $n=12 /$ group) and monitored as shown in Fig. 3a. MiRr-654 overexpression was confirmed before cell implantation, at 5 days postinjection and at the end of the experiment (Fig. 3b-d). Eight days post-injection, all miR-Control mice developed tumors whereas only $40 \%$ of miR-654 mice did so (Fig. 3e). Significant differences in tumor volume were visible at 11 days post-injection and maintained through the experiment (Fig. $3 \mathrm{f})$. Mice were sacrificed at day 25 and tumors were weighted and imaged, finding smaller tumors in the miR654 group (Fig. 3g, h). Importantly, miR-654 levels inversely correlated with tumor volume $(r=-0.95$, Fig. 3i) and weight $(r=-0.8619$, Supplementary Fig. 3a) and both positively correlated between each other, confirming the accuracy of the measures (Supplementary Fig. 3b).

Moreover, reduced cellularity, larger areas of collagen deposition and decreased percentage of Ki67 positive cells were visible in mice sacrificed at day 5 post-injection (Fig. $3 \mathrm{j}, \mathrm{k}$ ) and remained until the end of the experiment (Supplementary Fig. 3c); phenotype commonly observed after chemotherapy, where the extracellular matrix fuels the empty spaces left by death cells [25]. Of note, some tumors from the miR-Control group invaded the dermis, while none of the miR-654 mice group did so (Supplementary Fig. 3d). Altogether, miR-654 reduced tumor growth in vivo, inducing regressive changes at initial steps after the engraftment determining the tumor fate.

\section{MiR-654 targets multiple genes involved in apoptosis, cell proliferation and survival pathways}

To understand the molecular mechanisms regulated by miR654, we followed the workflow shown in Fig. 4a. First, a whole transcriptome 3'UTR search for miR-654 binding sites was conducted using the in silico platform miRWalk2.0 [26] that revealed a list of 2302 putative target genes. Of those, 46 were predicted by at least 5 different algorithms (Supplementary Table 11) and 14 were finally selected according to previously described functions related to the observed miR-654 phenotypes. Upon miR-654 overexpression 5 of those 14 candidates suffered a reduction in the mRNA levels in SKOV3 and A2780 cells with more than $50 \%$ reduction in at least one of them (Fig. 4b, red asterisks) (Supplementary Fig. 4a). At protein level, HAX1, RAB1B and PLAGL2 appeared decreased in both cell lines at different time points post-transfection, while CDCP1 and PBX3 protein levels did so only in SKOV3 
A
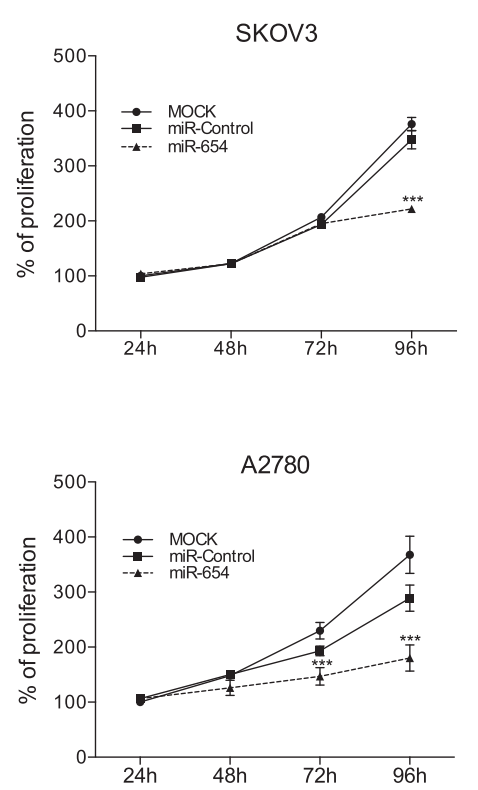

E

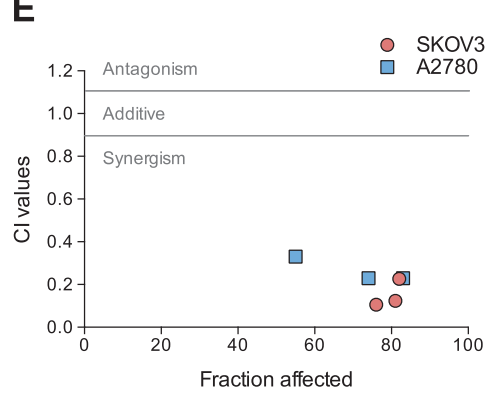

B
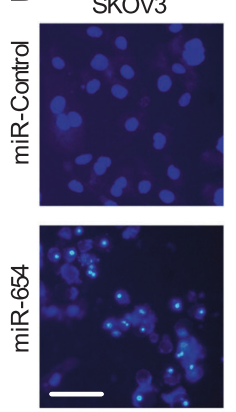

C

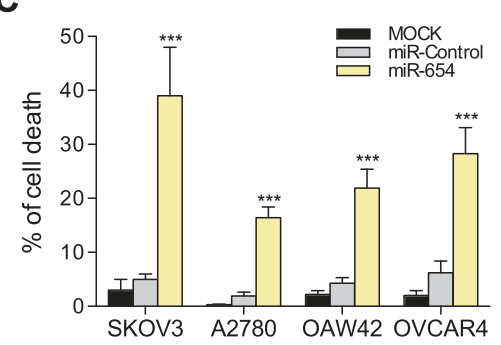

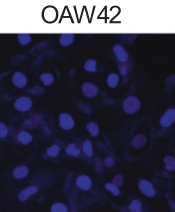
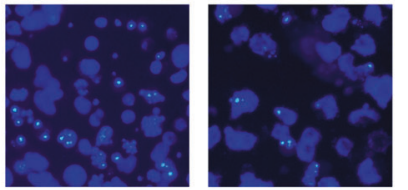

OVCAR4
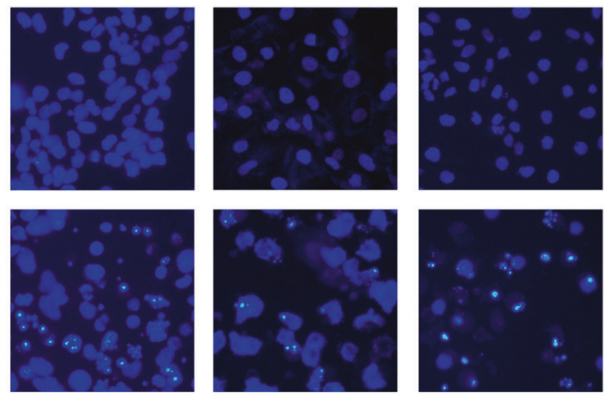

$\mathbf{F}$

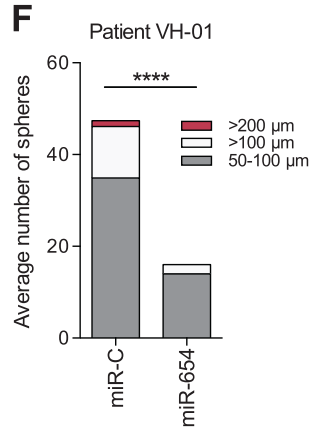

D

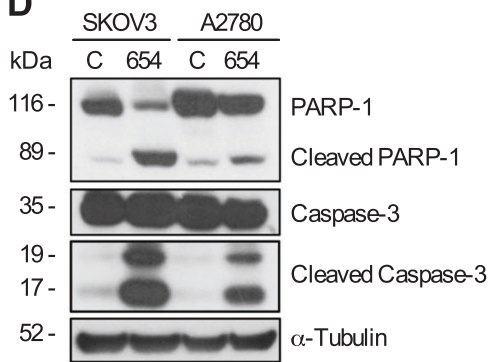

G
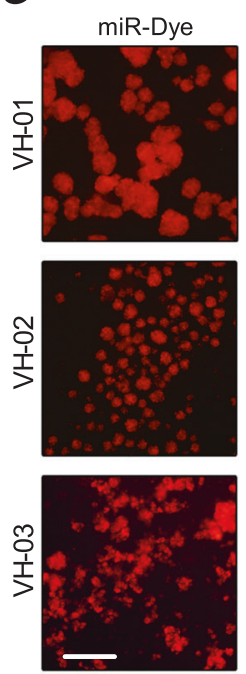
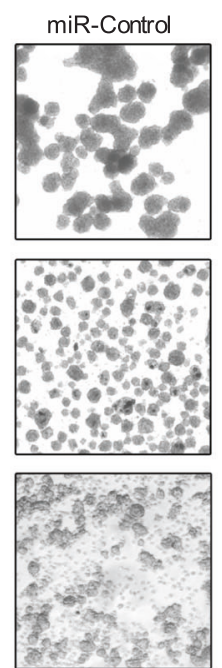
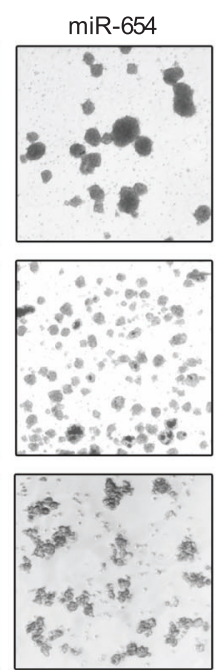

H
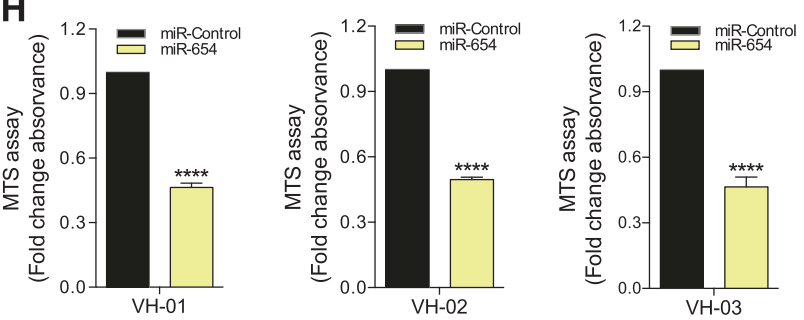

I

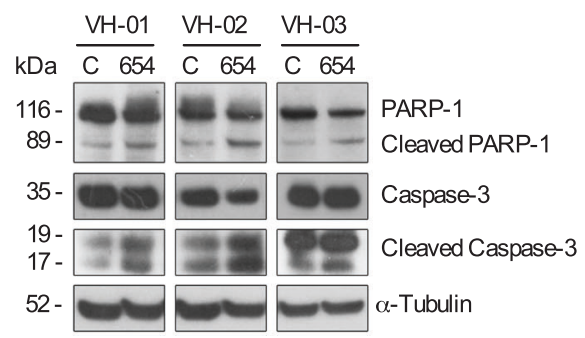

regions and this was significantly rescued when seed sequences in the $3^{\prime} \mathrm{UTR}$ regions of all targets were mutated (Fig. 4d, Supplementary Table 1k), indicating a direct modulation of these genes by miR- 654 . cells (Fig. 4c), possibly due to different protein stability between both cell lines. Furthermore, luciferase-reporter assays upon miR-654 overexpression showed a significant reduction in luciferase activity for all $3^{\prime} \mathrm{UTR}$ engineered
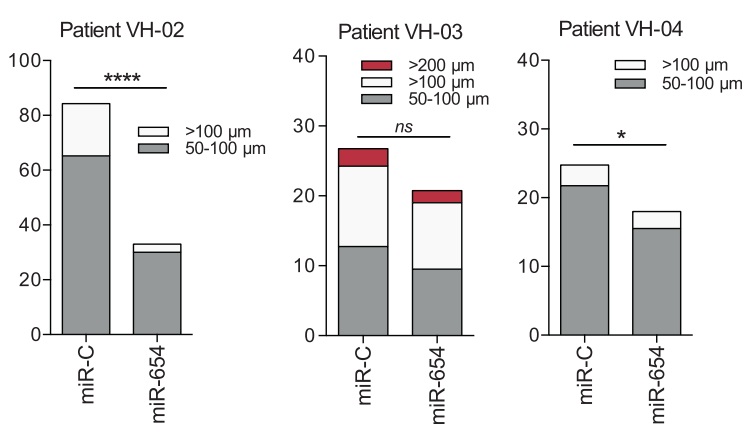
Fig. 2 MiR-654 expression reduces viability of OC cells and spheroids from patient-derived ascites, induces apoptosis and cooperates with paclitaxel to impair OC growth. a Time course proliferation assay in SKOV3 and A2780 cells after miR-654 or miR-Control transfection at $24,48,72$, and $96 \mathrm{~h}$ ( $n=5 /$ condition). b Representative images of the Hoechst nuclear staining upon overexpression of miR-654 or miRControl. Bar, $50 \mu \mathrm{m}$. $\mathbf{c}$ Quantification of B was performed based on all cells from 4 representative images $(n=3 /$ condition). d Western blot of PARP-1 and Caspase- 3 in SKOV3 and A2780 cells at $96 \mathrm{~h}$ posttransfection with miR-654 or miR-Control. e Combination index (CI) of miR-654 (constant, $25 \mathrm{nM}$ ) and paclitaxel at a $1 / 2 \times, 1 \times$ and $2 \times$ of the $\mathrm{IC}_{50}$ was calculated, by the Chou-Talalay method. DMSO and miR-Control were used as controls ( $n=6 /$ condition). Plotted data are CI values at the corresponding \% of fraction affected, when treated with different doses of paclitaxel for each cell line $\left(\mathrm{IC}_{50}=25.2\right.$ and $8.84 \mathrm{nM}$ for SKOV3 and A2780, respectively). * means comparison of each condition vs miR-Control/DMSO; \# Combo condition vs miR654/DMSO, +Combo condition vs the miR-Control/Paclitaxel. f The number of spheres was scored after $96 \mathrm{~h}$ post-transfection with miRControl and miR-654 in 4 OC patient-derived ascitic cells grown under anchorage independent conditions and classified as being between $50-100 \mu \mathrm{m}, \geq 100 \mu \mathrm{m}$, and $\geq 200 \mu \mathrm{m}$ in diameter. g Representative images of the indicated ascitic primary cells used for the sphere forming capacity assay. The miRNA-transfection control labeled with Dy547 was used to monitor transfection. Bar, $100 \mu \mathrm{m}$. h Viability assay (MTS) was performed at $96 \mathrm{~h}$ post-transfection with miRControl or miR-654 in the indicated OC patient-derived ascitic cells cultured under anchorage independent conditions. i Spheres were used for protein extraction and Western blot analysis with the indicated antibodies $96 \mathrm{~h}$ post-transfection with miR-Control and miR-654. Graph represents mean \pm SEM of three independent experiments in plots (a), (c), and (e). * ${ }^{\#},{ }^{+} p<0.05 ; * * p<0.01 ; * * * p<0.001$; **** $p<$ 0.0001 ; $n s$ not significant. $p$ values were calculated using unpaired student's $t$-test

\section{CDCP1 and PLAGL2 knockdown phenocopies miR- 654 overexpression in vitro}

To gain insights into the molecular mechanisms driven by miR-654 through its targets, HAX1, RAB1B, PBX3, CDCP1 or PLAGL2 were silenced in SKOV3 cells (Supplementary Fig. 4b). CDCP1 and PLAGL2 silencing best recapitulated the effects of miR-654 overexpression, reducing proliferation (Fig. 4e) and increasing the percentage of apoptotic cells comparably to miR-654 overexpression (Fig. $4 \mathrm{f}, \mathrm{g})$. In addition, overexpression of miR-654 in the ex vivo patient-derived ascitic cell models resulted in diminished protein levels of CDCP1, PLAGL2 and RAB1B (Fig. 4h), thereby suggesting that these 3 genes could be mediators of the miR-654 effects in this context. Interestingly, we found higher $C D C P 1$ and PLAGL2 levels in HGSC OC samples compared to benign lesions (Fig. 4i), while $R A B 1 B$ levels were similar between groups (Supplementary Fig. 4c). We found that CDCP1 or PLAGL2 and miR-654 levels inversely correlated (although did not reach statistically significance for PLAGL2 and miR-654) (Supplementary Fig. $4 d)$. This suggests that CDCP1 correlates better with miR654 levels and the clinical parameters, indicating a close relation of miR-654/CDCP1 with OC progression. Instead, the lack of significant correlation between PLAGL2 and miR-654 in individual patients might indicate a more complex regulation of this target. Together, these results support the clinical relevance of targeting CDCP1 and PLAGL2 by miR-654 in OC.

\section{MiR-654-CDCP1/PLAGL2 converge on the MYC, AKT and Wnt gene sets}

For the abovementioned reasons, we focused on understanding CDCP1 and PLAGL2 signaling networks regulated by miR-654 hereafter. To this end, a whole transcriptome analysis was performed after silencing CDCP1 or PLAGL2 in SKOV3 cells (Fig. 5a). Principal component analysis (PCA) segregated siControl vs. siCDCP1 or siPLAGL2 samples indicating a consistent transcriptional impact after both gene depletions (Fig. 5b), and a high number of differential expressed genes (Fig. 5c, FDR $<0.25$ and fold change $> \pm 1.5$ ). Interestingly, 70 and 60 genes were commonly down and upregulated, respectively, by CDCP1 and PLAGL2 silencing (Fig. 5d), denoting that part of the transcriptional changes partially overlapped between both genes. Functional annotation using DAVID bioinformatic tools showed that CDCP1 and PLAGL2 depletions significantly impacted on genes associated to canonical KEGG pathways in cancer (Fig. 5e, $>10$ genes and $p<0.05$ ), and to GO terms related to cell proliferation and apoptotic processes, among others (Supplementary Fig. 5a). Furthermore, gene set enrichment analysis (GSEA) was performed using major gene set collections, resulting in a vast number of significantly enriched gene sets for both gene conditions (Supplementary Fig. 5b). Then, gene sets related to apoptosis, HIF-1, focal adhesion, Wnt, MYC, actin cytoskeleton, MAPK, AKT, FoxO, Proteoglycan in cancer, and angiogenesis were used for a second GSEA, according to the most represented functions found in the DAVID analyses and the first GSEA. Noticeably, several enriched gene sets related to MYC, AKT and Wnt pathway were commonly altered by CDCP1 and PLAGL2 knockdown, among others (Fig. 5f-h, Supplementary Fig. 5c, and Supplementary Methods). MYC levels appeared downregulated by both CDCP1 and PLAGL2 depletions (Fig. 5h, top left), as well as several regulators of the Wnt pathway such SMURF2 and DKK1 (Fig. 5h, top right). Additionally, several cyclin-dependent kinases (CDKs) such as CDK2 and CDK4 diminished upon PLAGL2 depletion (Fig. 5h, bottom right), as well as the levels of $A K T 2$ and retinoblastoma (RB) (Fig. 5h, bottom right), thus suggesting that reduction of the expression of these genes might contribute to the effects of miR-654 overexpression on cell survival. In agreement with the hypothesis that part of the miR-654 phenotypic effects occur through the reduction of CDCP1 and PLAGL2 levels 
A

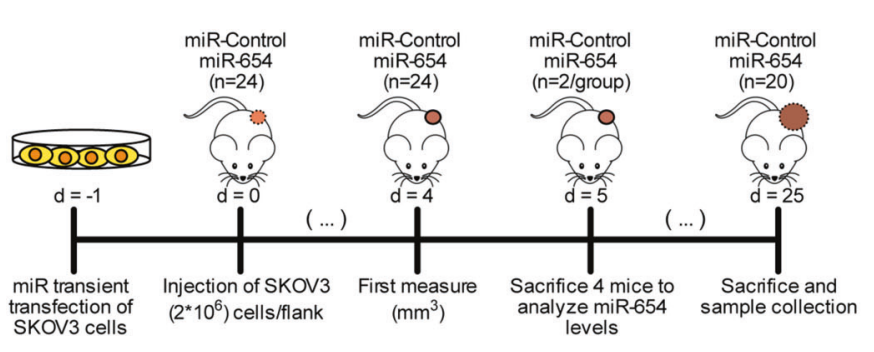

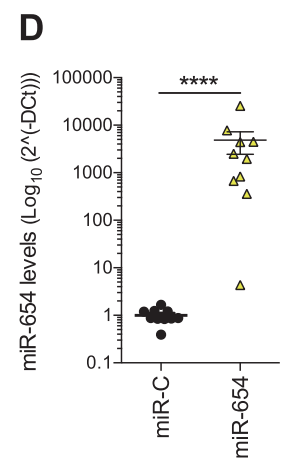

E

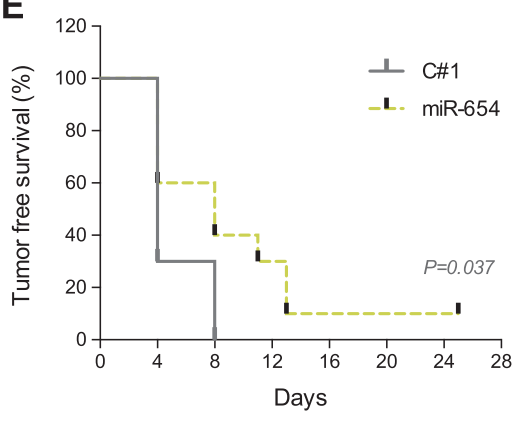

B

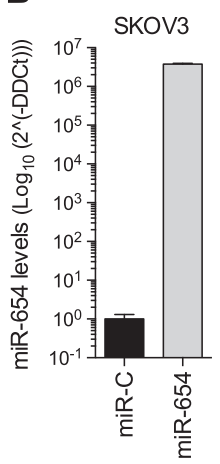

C

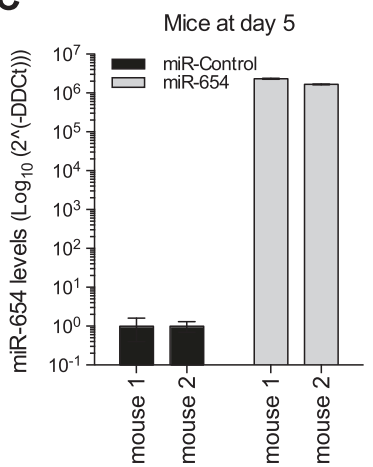

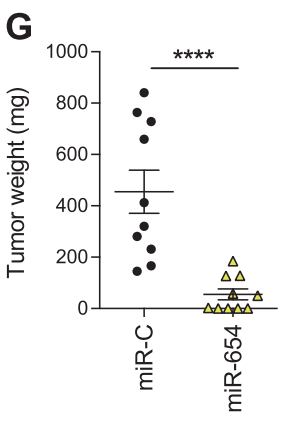

J


F

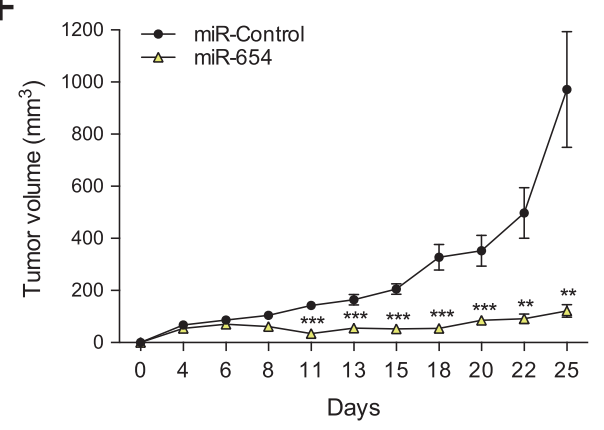

H

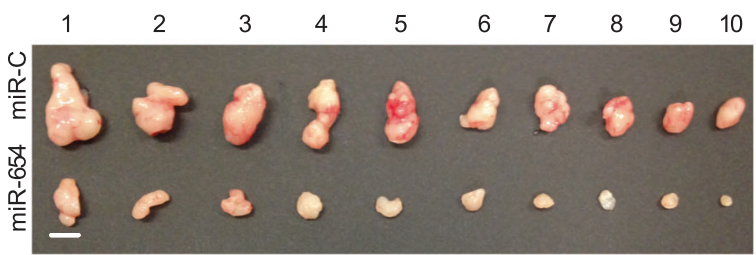

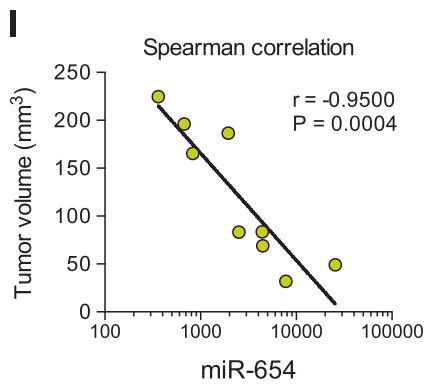

in $\mathrm{OC}$, we found diminished protein levels of $\mathrm{MYC}$, $\mathrm{p}$ AKT, CDK2/4, RB, p-RB and $\beta$-catenin ( $\beta$-cat, gene symbol $C T N N B 1$ ), and increased levels of p27 (also $C D K N 1 B$ )
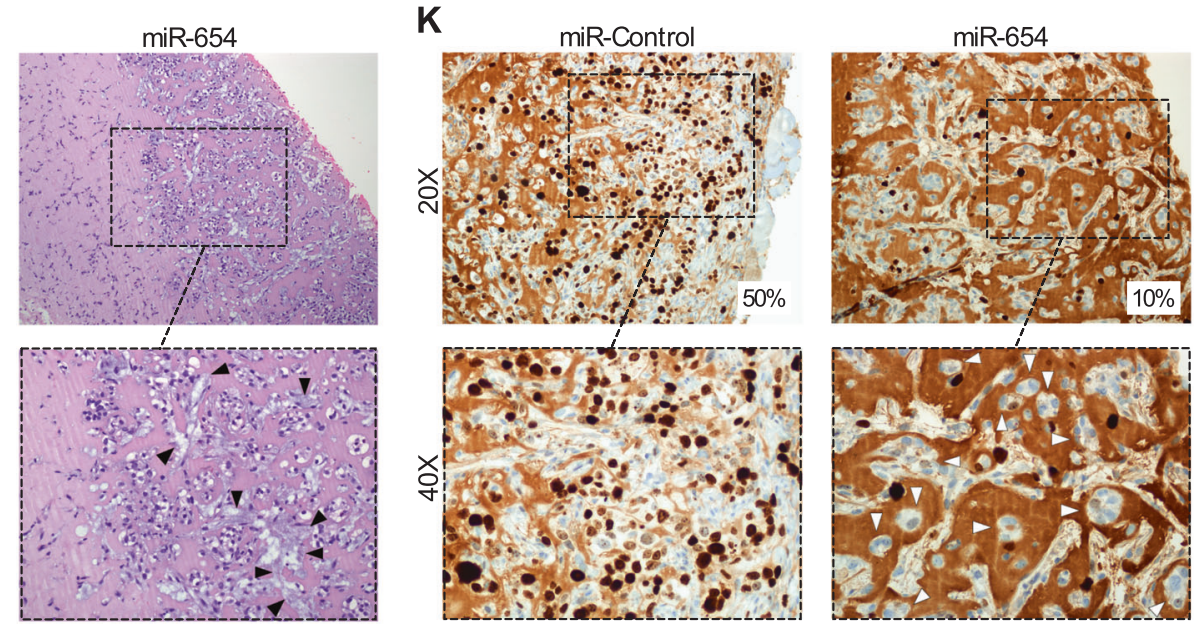

upon overexpression of miR-654 in SKOV3 cells (Fig. 5i), confirming the expected molecular events that accompany a slower cell cycle progression and a decreased cell survival. 
Fig. 3 Overexpression of miR-654 impairs tumor growth in vivo. a Schematic representation of the in vivo model timeline. b-d Relative expression of miR-654 levels analyzed by RTqPCR in SKOV3 cells before mice injection (b), in two mice per group at day 5 post-injection (c), and in the 20 mice xenografts at the end-point of the experiment (d). e Tumor-free survival curve of mice bearing OC xenografts transfected with miR-Control (continuous line) or miR-654 (dotted line). f Tumor volume of mice injected with either miR-Control or miR-654 transfected SKOV3 cells $(n=10$ /group), measured for 25 days. g Weight of resected tumors. h Macroscopic images of resected tumors at the consecution of the experiment. Bar: $1 \mathrm{~cm}$. i Spearman correlation between miR-654 levels and tumor volume of miR-654 mice group. $\mathbf{j}$ Representative images of H\&E staining of the OC xenografts after 5 days post-injection. Black arrows are showing areas of collagen deposition by the reduced tumor cellularity in miR654 tumors. k Representative microscopic images of Ki67 staining of the OC xenografts after 5 days post-injection. White arrows are showing remaining areas of non-proliferating tumor cells (without Ki67 staining). $* * p<0.01$; ***p $p<0.001 ; * * * * p<0.0001 . p$ values were calculated using unpaired student's $t$-test for $(\mathbf{d}),(\mathbf{e}),(\mathbf{f}),(\mathbf{g})$

We also sought to explore whether these pathways were modulated by miR-654 in the patient-derived ascitic cell models and, concurring with our previous results, the protein levels of MYC, p-AKT, CDK2/4 and $\beta$-cat were diminished upon miR-654 overexpression in patient-derived ascites (Fig. 5j). Altogether, these results suggested that the tumor suppressor functions of miR-654 are mediated, in part, by the modulation of its direct targets, CDCP1 and PLAGL2, which in turn modulate key pathways in OC, such as MYC, AKT and Wnt pathways (Fig. 6).

\section{Discussion}

The clinical management of OC remains a challenge due to the fact that high-grade histologies generally become resistant to standard therapies [3]. Moreover, the ability to translate the increasing knowledge on the genetic alterations into more effective treatments for OC had, so far, limited clinical impact in part due to the heterogeneity of the disease. In the present study, we found 4 miRNA whose levels were higher in tumors from SSV OC patients, suggesting at first glance an oncogenic role for them. However, the restoration of miR-127-3p and miR-654 reduced cell proliferation, evoking tumor suppressive rather than oncogenic properties. A plausible explanation is that SSV tumors could be more infiltrated with certain cell types from the immune system that have been associated to poorer prognosis in OC [27], which may contribute to the increased levels of miR-654 found in SSV compared to LSV tumors (our preliminary results). In agreement with the previous phenotype, we found higher miR-654 levels in normal samples than in OC tumors, which was also seen for miR$127-3 p$ [28], suggesting that their loss may contribute to the initiation of OC.
In accordance with the in vitro results, we proved for the first time that the ectopic expression of miR-654 reduced tumor growth in vivo and reduced viability of patientderived ascites grown ex vivo. Importantly, this is the first report that tested the effect of modulating a tumor suppressor miRNA on the sphere forming capacity and viability of OC patient-derived ascites. This model is not without limitations, as their use is limited to 4-6 passages [29], but our results clearly suggest that the tumor-suppressive effects of miR-654 could be of clinical relevance. Nonetheless, other hits presumably accompany miR-654 loss in OC cell transformation, as inhibition of miR-654 in non-tumoral immortalized ovarian surface epithelial (IOSE) cells per se, does not impair the cell death by contact inhibition typically observed in these cells (not shown).

MiR-654 was firstly identified and annotated by Cummins JM et al. [30] in 2006, it is located in the $14 \mathrm{q} 32$ chromosomal region, and recently found downregulated in classic Hodgkin lymphoma [31] and breast cancer [32]. Here, we found that the $14 \mathrm{q} 32$ region is hypermethylated in human OC tissues and cell lines, and an increased expression of miR-654 was obtained upon DNA methyltransferase inhibition in vitro, suggesting that epigenetic silencing could be one of the possible mechanisms of miR-654 regulation. Concurring with this observation, the DLK1-MEG3 (Dlkl-Gtl2) cluster, which contains miR-654 in humans [33], has been found epigenetically downregulated in OC through hypermethylation [13].

To date, little is known about the downstream miR-654dependent signaling, and EPSTI1 for breast [32] and AR for prostate [34] cancer were the only 2 direct targets identified thus far. In this study, we found HAX1, RAB1B, PBX3, CDCP1 and PLAGL2 to be direct miR-654 targets, and their silencing revealed that the miR-654-related phenotype in OC is primarily caused by direct targeting of CDCP1 and PLAGL2 oncogenes. Furthermore, CDCP1 and PLAGL2 levels were found to be increased in ovarian tumors compared to normal samples, which has also been seen in leukemia [35] for PLAGL2, and in other malignancies including OC $[36,37]$ for CDCP1, supporting their role in OC progression and turning them into attractive therapeutic targets.

Our transcriptomic analysis after CDCP1 and PLAGL2 depletion defined both genes as master regulators of MYC, AKT and Wnt pathway in OC cells, and several of the canonical genes altered at the transcriptome level were confirmed at protein level in OC cell and primary patientderived ascites. CDCP1 (CUB domain-containing protein 1) is a type I transmembrane glycoprotein, that activates cell survival pathways in $\mathrm{OC}$ to mediate spheroid formation and chemoresistance through the activation of $p$ the AKT pathway $[36,37]$. PLAGL2 belongs to the PLAG family of zing finger proteins [38] and acts as an oncogene by enhancing anchorage independent growth and inducing 
A

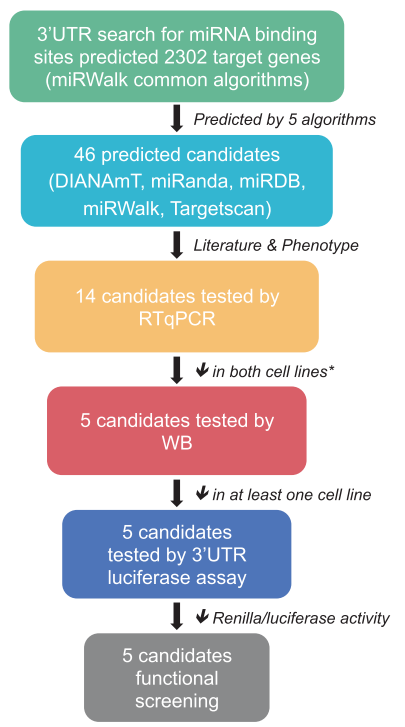

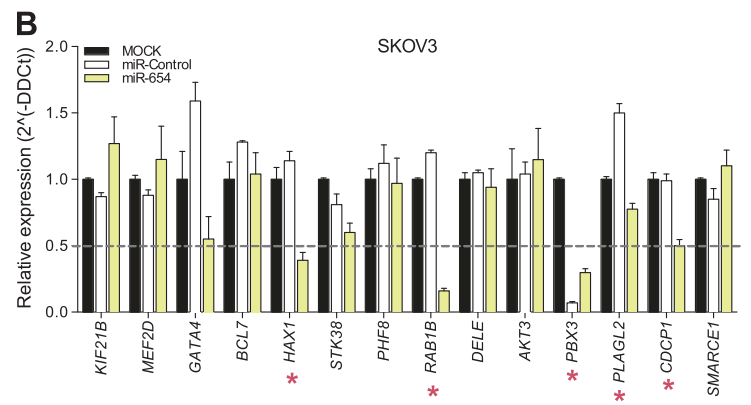

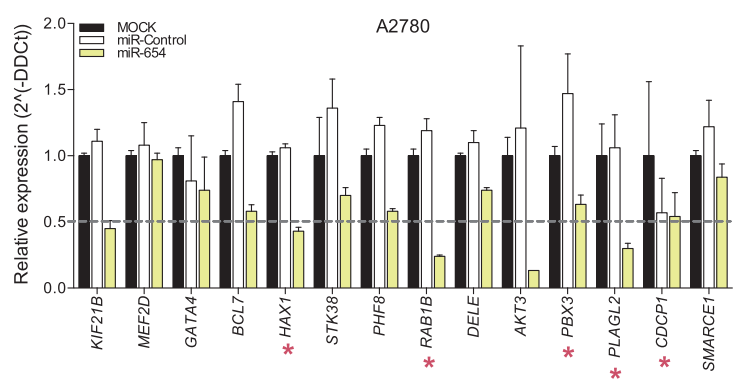

C

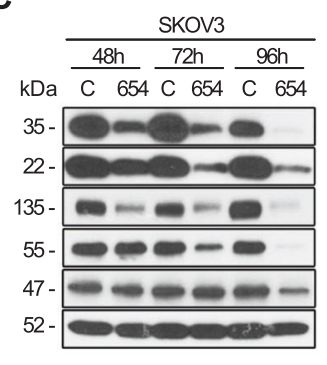

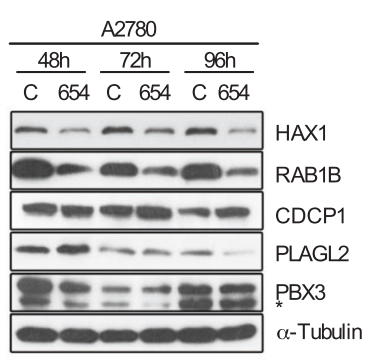

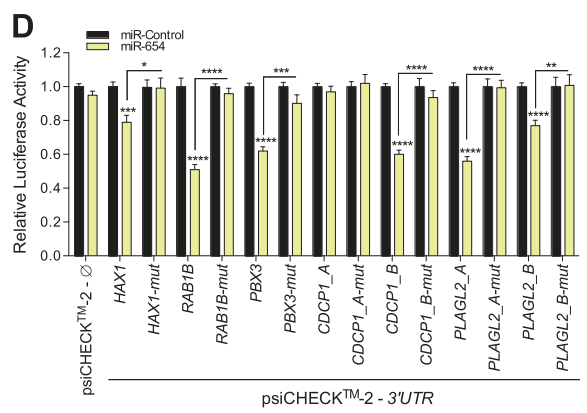

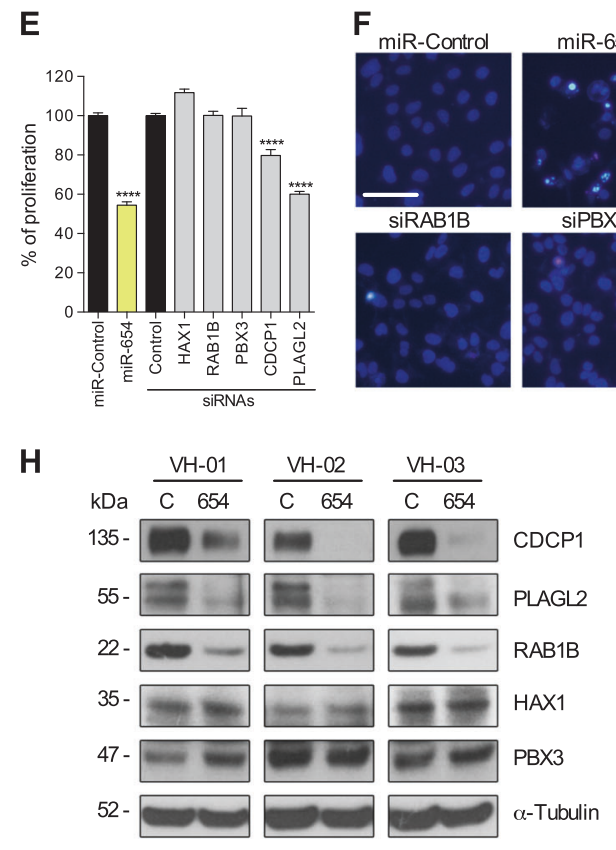

E

tumors in mice [38], in part through its downstream effector p-AKT [39]. Here, we found reduced AKT2 mRNA levels by PLAGL2 inhibition, which together with the reduced
I
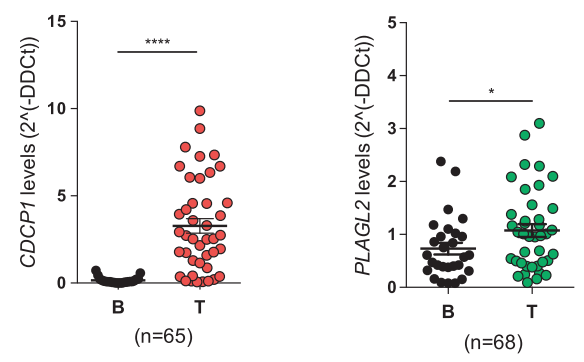

p-AKT levels observed upon miR-654 overexpression, suggest a possible contribution of CDCP1 and PLAGL2 to the regulation of AKT pathway through miR-654 signaling. 
Fig. 4 MiR-654 targets multiple cancer-related genes involved in apoptosis, cell proliferation and survival pathways, unveiling CDCP1 and PLAGL2 as relevant direct targets in OC. a Schematic representation of the workflow followed to find miR-654 target genes. b Relative mRNA expression levels of the indicated putative miR-654 target genes at $48 \mathrm{~h}$ post-transfection with miR-654, miR-Control or MOCK-non-transfected SKOV3 (top panel) and A2780 (bottom panel) cells. Red asterisks point to the putative miR-654 targets selected for protein level determination. c Representative Western blot for the indicated proteins at 48,72 and $96 \mathrm{~h}$ post-transfection in SKOV3 (left panel) and A2780 (right panel) cells. Asterisk points at the specific band. d Luciferase assay performed in HEK-293T cells cotransfected with the indicated luciferase-reporter vector (corresponding WT and MUT 3'UTR regions for each target, respectively) and miR654 or miR-Control mimics. e Proliferation assay after individual silencing of the miR-654 targets genes ( $n=6 /$ condition). f Representative images of the Hoechst nuclear staining upon transfection with miR-654 or miR-Control mimics, siR-Control or the corresponding siRNA for each of the target genes at $96 \mathrm{~h}$ post-transfection of SKOV3 cell line. Bar, $50 \mu \mathrm{m}$. $\mathrm{g}$ Quantification of $\mathrm{F}$ from four representative images $(n=3 /$ condition). $\mathbf{h}$ Western blot of the indicated miR-654 targets in three patient-derived ascitic cells from OC patients transfected with miR-Control or miR-654 for $96 \mathrm{~h}$. i Relative mRNA expression of $C D C P 1$ and PLAGL2 analyzed in human ovarian tissues $(n=65$ and $n=68$, respectively, according to the outlier samples detected for each gene and including only HGSC tumors). $b$ benign lesions, $T$ tumors. Graphs represent the mean \pm SEM of four independent experiments for (d), (e), and (g). ${ }^{*} p<0.05$; $* * p<$ $0.01 ; * * * p<0.001$; ****p $<0.0001$. $P$ values were calculated using unpaired student's $t$-test for (d), (e) and (g), and non-parametric MannWhitney test for (i)

A striking decrease in $\mathrm{p}-\mathrm{RB}$ was also observed upon miR-654 expression, possibly caused by the reduced $\mathrm{p}$ AKT and CDK2/4 levels observed. In addition, 2 CDK regulatory proteins, $C K S 1 B$ and $\mathrm{p} 27$, were found under and overexpressed, respectively, upon both CDCP1 and PLAGL2 inhibition, suggesting that cell cycle progression and survival was diminished in OC cells in part through defective CDKs function.

Importantly, this is the first report that relates $\mathrm{CDCP} 1$ and PLAGL2 with MYC, a master regulator of cancer cells [40]. $M Y C$ has been found to be mutated and amplified in HGSC $[3,16]$ and a good candidate for targeting platinum-resistant OC [41], thereby suggesting that these patients would likely benefit of a miR-654-based therapy. Furthermore, several genes related to the Wnt pathway were found to be altered after CDCP1 and PLAGL2 depletion in OC cells, and reduced total $\beta$-cat protein levels were observed upon miR654 overexpression in OC cells and primary ascites confirming the modulation of the Wnt pathway by miR-654. Whereas PLAGL2 has been shown to activate Wnt signaling in glioblastoma and colon cancer $[42,43]$ thereby resulting in an accumulation of total $\beta$-cat levels, CDCP1 had never been previously related to the Wnt pathway, which further supports the use of miR-654 as a potent Wnt-like inhibitor, through targeting CDCP1/PLAGL2.

In summary, miRNA-654 acts transversally against multiple pathways, including MYC, AKT and Wnt signaling in OC, reducing cell proliferation and promoting cell death (Fig. 6). We found it downregulated in ovarian tumors, together with elevated levels of its direct targets CDCP1 and PLAGL2, which might contribute on boosting OC cells tumorigenicity. In summary, these results pinpoint that miR-654 restoration may be an effective strategy for the treatment of $\mathrm{OC}$ in combination with the current therapies.

\section{Materials and methods}

\section{Human samples}

Formalin-fixed paraffin embedded (FFPE) primary tumors from 107 advanced stage OC patients were obtained from Vall Hebron University Hospital (VHUH) (Barcelona, Spain) and 5 other national collaborative hospitals (Supplementary Table 1a) used for miRNA array analyses. Fresh tissues including benign lesions and ovarian tumors (Supplementary Table $1 \mathrm{~b}$ ), and 21 patient-matched paired FFPE tumors and metastases were obtained from VHUH (Supplementary Table 1c). All patients gave their written informed consent, and fresh tissues were collected conforming to the Institutional Review Board (IRB) (PRAMI3082015).

\section{Cell culture}

Commercial cell lines, SKOV3, OAW42, 59 M, OAW28, OVCAR4, TOV112, OV90, A2780, A2780cis, BIN67, IGROV1, IOSE-503, IOSE-385 and HEK293T were cultured with indicated culture media (Supplementary Table 1d). Cells were regularly tested for mycoplasma contamination and authenticated by short tandem repeat profiling.

For patient-derived ascites primary cultures, cells were obtained from malignant ascitic fluid collected at the time of the surgery under the IRB approval, (Supplementary Table 1e). Ascitic primary cultures were established following procedures previously described [29]. Immediately after surgery, the ascitic fluid was mixed (1:1) with Mix medium (Supplementary Table 1e). After 1 week, medium was replaced to remove any remaining blood cells and adherent cells were grown until 4-6 passages. All cells were maintained at $37{ }^{\circ} \mathrm{C}$ in a saturated atmosphere of $95 \%$ air and $5 \% \mathrm{CO}_{2}$.

\section{Human miRNA microarray}

For miRNA expression profiling, TaqMan ${ }^{\circledR}$ Array Human MicroRNA A + B Cards Set v3.0 (Applied Biosystems, CA, USA) were used to analyze a total of 768 miRNA sequences by $2^{-\Delta \Delta \mathrm{Ct}}$ method [44]. Samples from $27 \mathrm{FFPE}$ tumors (Supplementary Table 1a) were used for the discovery phase. Total RNA was used for the reverse 
A

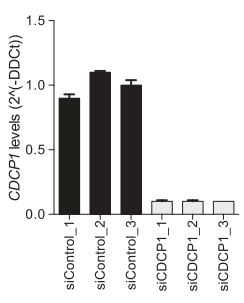

D

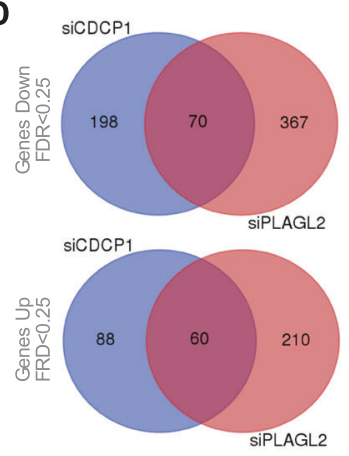

$\mathbf{F}$

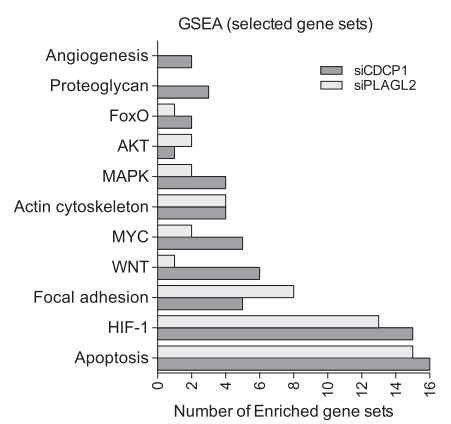

H

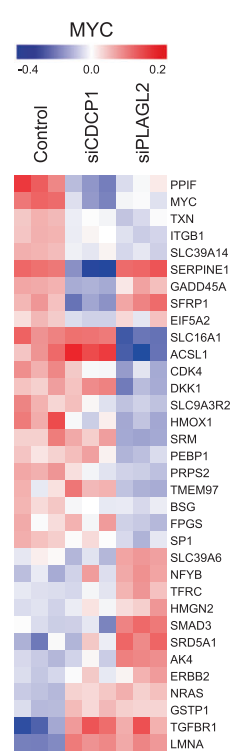

E
B

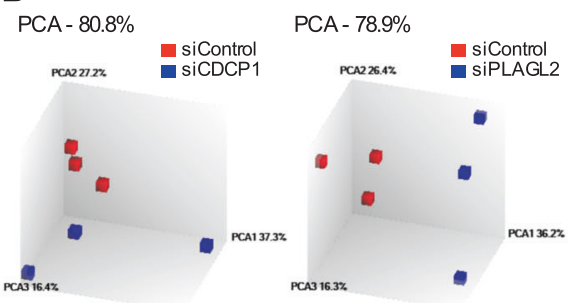

C
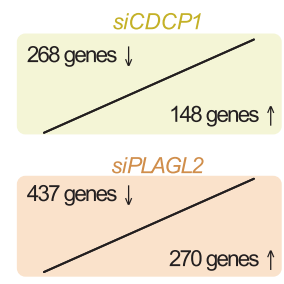
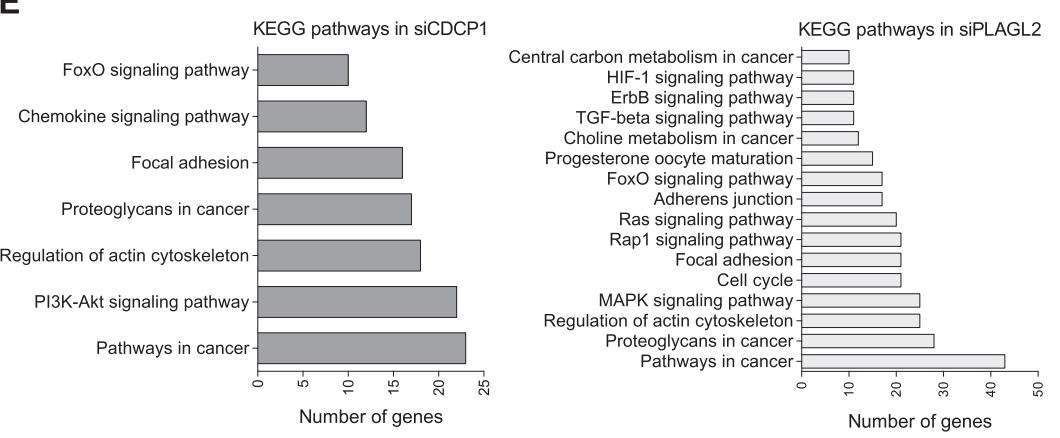

G

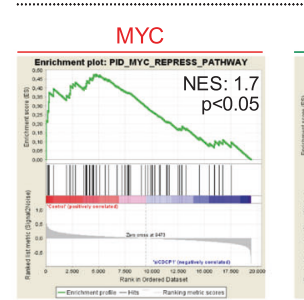

GSEA of siCDCP1 cells
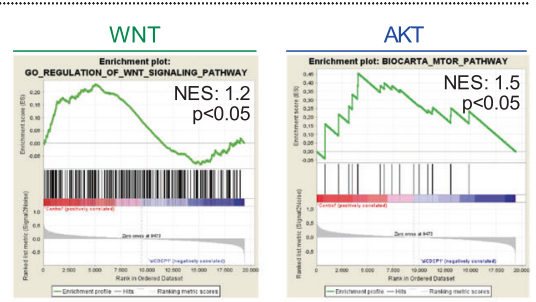

GSEA of SiPLAGL2 cells
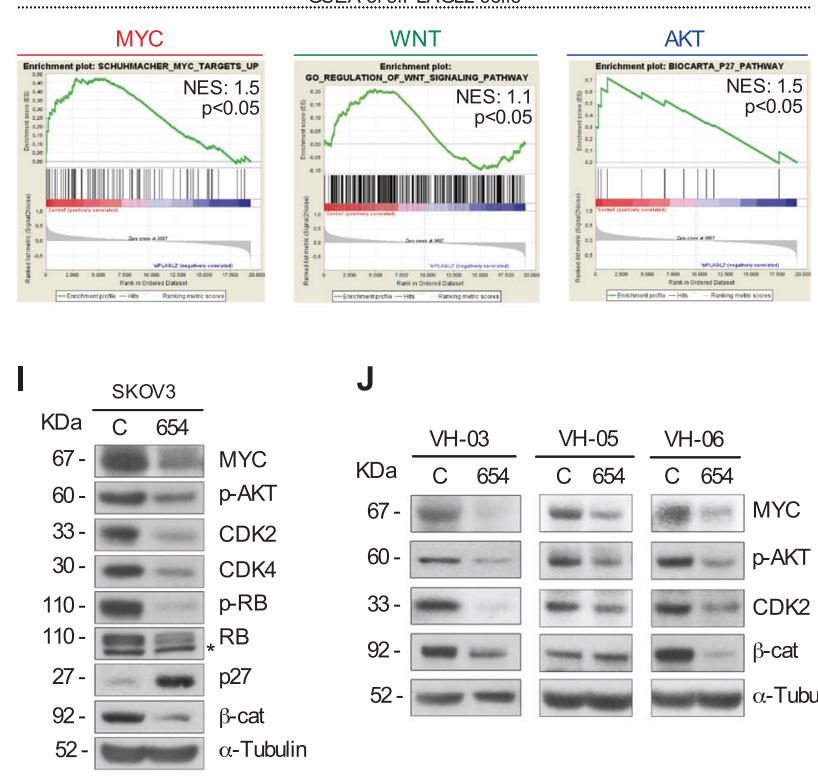

J

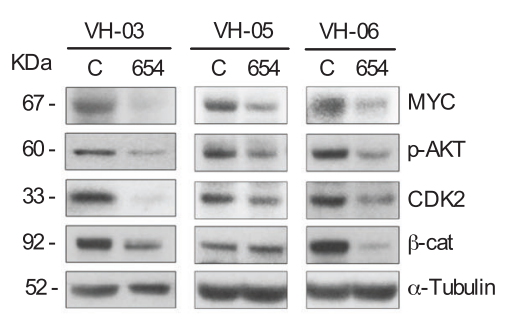

transcription (RT) of $300 \mathrm{ng}$ by using TaqMan MicroRNA RT Kit and the pool Set v3.0 of Megaplex ${ }^{\mathrm{TM}}$ RT Primers A +B (Applied Biosystems, CA, USA). Targeted preamplified cDNA was obtained by using MegaplexTM PreAmp Primers, Human Pool Set v3.0 A + B and TaqMan ${ }^{\circledR}$ PreAmp Master Mix $2 \times$ (Applied Biosystems, CA, 
Fig. 5 miR-654 impacts on several oncogenic pathways through PLAGL2 and CDCP1 in OC. a Levels of CDCP1 and PLAGL2 depletions analyzed at mRNA level before microarray analysis was performed in SKOV3 cells ( $n=3 /$ condition). b PCA was performed by Affimetrix Expression Console software to show segregation of distinct expression profiles for siCDCP1 (left) and siPLAGL2 (right) $v s$. siControl cells. c Summary of the deregulated genes upon CDCP1 and PLAGL2 silencing (FDR $<0.05$ and fold change $> \pm 1.5$ ). d Venn diagram with downregulated (top) and upregulated (bottom) genes upon CDCP1 and PLAGL2 silencing compared to siControl cells (FDR $<0.25$ and fold change $> \pm 1.5$ ). e KEGG pathway analysis by DAVID bioinformatic tool, using differentially expressed genes with FDR $<0.05$ from CDCP1 (left) and PLAGL2 (right) depletions compared to siControl cells. f GSEA: gene sets collections related to the indicated processes were downloaded from MSigDB.v6 and used for the analyses. Total number of enriched gene sets with FDR $<0.25$ or $p<$ 0.05 were plotted regarding each collection and gene depletion. $\mathbf{g}$ Representative GSEA curves for significant enriched gene sets related to MYC, Wnt and AKT pathways upon CDCP1 and PLAGL2 silencing compared to siControl cells. Corresponding normalized enriched score (NES) and $p$-values $<0.05$ for enriched gene sets are included. h Heatmap depicting deregulated genes upon CDCP1 and/or PLAGL2 depletion with FDR $<0.05$ and fold change $>$ \pm 1.5 , which are found in the collapsed gene lists of the enriched gene sets for each pathway. $\mathbf{i}$ Western blots of the indicated proteins to verify the alteration in MYC, AKT and Wnt signaling upon miR-654 overexpression in SKOV3 cells at $96 \mathrm{~h}$ post-transfection. Tubulin was used as a loading control. $\mathbf{j}$ Western blot analyses in spheroids from patient-derived ascitic cells collected after $96 \mathrm{~h}$ post-miRNA transfection to verify the alteration in MYC, AKT and Wnt signaling in human samples ex vivo

USA). Two miRNA array cards were performed per patient in a 7900 ABIPrism System (27 cards A, 27 cards B). Custom RT and Preamp primer pools were used to validate the 32 differentially expressed miRNAs in 80 FFPE tumors (Supplementary Table 1a), by using Custom TaqMan ${ }^{\circledR}$ Array MicroRNA Cards following the manufacture instructions.

\section{RTqPCR}

For miRNA analyses, $25 \mathrm{ng}$ of total RNA was used for RT of each individual miRNA, by using specific TaqMan probes (Supplementary Table 1f). Hsa-miR-572 was used as endogenous control. For mRNA analyses, $1 \mu \mathrm{g}$ of total RNA was used for RT using SuperScript III enzyme (Invitrogen). Power SybrGreen Master MIX (Cat\# 4367659) was used to analyze mRNA levels by the corresponding designed primers (Supplementary Table 1g). GAPDH was used as endogenous control. Human samples were randomized for RNA extractions, RT reactions and RTqPCR analyses in all cases. Relative quantification of gene expression was performed with the $2^{-\Delta \Delta \mathrm{Ct}}$ method [44].

\section{DNA methylation microarray}

DNA methylation data was obtained from Illumina Infinium Human Methylation450 BeadChip arrays, and was downloaded from the TCGA portal [16], GSE81224 dataset [45], and the Cancer Epigenetics and Biology Program (PEBC) dataset (unpublished) for OC primary tumors $(n=35)$; and from the GSE68379 dataset [46] for OC cell lines $(n=43)$. Briefly, the GenomeStudio 2010.3, Methylation Module 1.8.5 was used as previously described [47] to calculate the methylation $\beta$-values of the $13 \mathrm{CpG}$ sites of the miR-654 chromosomal region $14 \mathrm{q} 32.1$. The percentage of samples being hypermethylated was obtained by the average $\beta$-value of the $13 \mathrm{CpG}$ sites.

\section{DNA demethylating assay}

OAW28 and A2780 cells were treated with DMSO or the DNA methyltransferase inhibitor 5'AZA-2'desoxycytidine (Sigma). At the indicated time points, cells were used for miR-654 evaluation. MEG3 (Chr.14), GBGT1 (Chr.9) and MAGE-A3 (Chr.X) genes were used as positive control.

\section{Cell proliferation assay}

Cells were reversely transfected with $25 \mathrm{nM}$ of miRIDIAN miRNA mimic Negative Control, miRNA mimics or antagomiRs (Supplementary Table $1 \mathrm{~h}$ ), or with $50 \mathrm{nM}$ of the indicated siRNAs (Supplementary Table 1i) using Lipofectamine $^{\circledR} 2000$ and seeded at $3 \cdot 10^{3}-10 \cdot 10^{3}$ in 96 -well plates $(n=6 /$ condition). At the indicated time points, cells were fixed with $4 \%$ formaldehyde, stained with $0.5 \%$ crystal violet, dissolved with $15 \%$ acetic acid and measured at $590 \mathrm{~nm}$.

\section{Protein extraction and western blot}

Cells were harvested in RIPA buffer (Tris $\mathrm{HCl} 1.5 \mathrm{M} \mathrm{pH}=$ $8.8, \mathrm{NaCl} 5 \mathrm{M}$, Triton X-100, EDTA $500 \mathrm{mM}$ ) with protease and phosphatase cocktail inhibitors (1183170001, P5726, P0044, Sigma). Lysis was done on ice for $1 \mathrm{~h}$ with $30 \mathrm{sec}$ vortex $/ 15 \mathrm{~min}$. Proteins (20-80 ug) were resolved on $8-15 \%$ tris-glycine PAGE or pre-cast electrophoresis gels (Thermo Fisher), and transferred onto PVDF membranes, which were blocked for $1 \mathrm{~h}$ with $5 \%$ non-fat milk (Panreac) or 5\% BSA (Sigma) and probed overnight at $4{ }^{\circ} \mathrm{C}$ with the indicated antibodies (Supplementary Table $1 \mathrm{j}$ ). After $1 \mathrm{~h}$ incubation with horseradish peroxidase-conjugated secondary antibodies signal was acquired with Immobilon Western (Millipore) or ECL (GE Healthcare).

\section{Cell death assay}

Cells were reversely transfected with Negative Control or miR-654 mimics $(25 \mathrm{nM})$, or with the indicated siRNAs $(50 \mathrm{nM})$ and plated in 24 -well plates $\left(15 \times 10^{3}-10 \times 10^{4}\right.$ cells/well) and $96 \mathrm{~h}$ post-transfection cell death was 

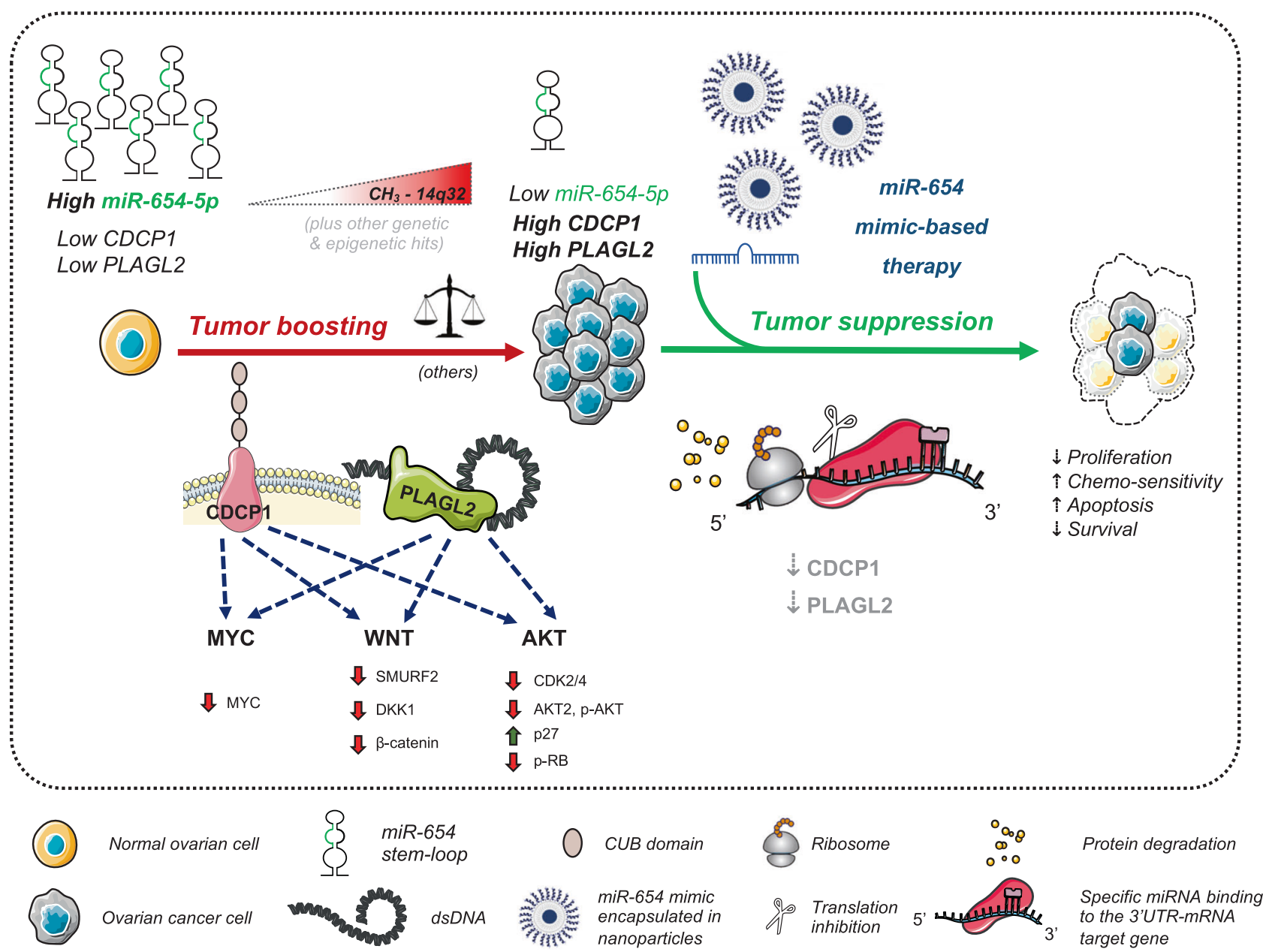

Fig. 6 Functions and model of the tumor suppressor role of miR-654 in OC. Proposed model of the tumor suppressor role of miR-654 in OC. The high levels of hypermethylation could partly explain the low miR-654 levels found in found in OC tumors and cells lines, which accompanied by the enhanced CDCP1 and PLAGL2 levels might contribute to boost the tumorigenicity of OC cells. Furthermore, PLAGL2 and CDCP1 oncogenes appeared as relevant direct targets of miR-654 in OC since they mediate at least part of the miR-654 function in the studied models. In addition, CDCP1 and PLAGL2

evaluated as previously reported [48]. Briefly, cells were stained with $0.05 \mathrm{mg} / \mathrm{mL}$ Hoechst 33258 and those with uniformly stained chromatin or with fragmented and/or condensed chromatin were scored as healthy or apoptotic, respectively. Similarly, cell death was evaluated upon caspase inhibition by using Q/VD/OPH at $10 \mu \mathrm{M}$ in SKOV3 cells reversely transfected with Negative Control or miR654 mimics $(25 \mathrm{nM})$ at $96 \mathrm{~h}$ post-transfection.

\section{Combination index analysis}

SKOV3 and A2780 cells transfected with Negative Control or miR-654 mimics ( $25 \mathrm{nM}$ ) for $48 \mathrm{~h}$ were incubated with paclitaxel for another $48 \mathrm{~h}$ at the indicated doses. impact on key cell regulators (red or green for genes found over or underexpressed upon CDPC1 and/or PLAG2 depletion and miR-654 overexpression), such as AKT, MYC and Wnt signaling pathways. Therefore, we show that overexpression of miRNA-654 reduces cell proliferation, induces apoptosis and sensitizes OC cells to paclitaxel addition. Together, our results suggest that restoration of miRNA-654 levels and/or co-targeting of PLAGL2 and CDCP1 oncogenes may be an efficient strategy for OC treatment in combination with the current therapies

Combination index were determined by the Chou-Talalay method using the Compusyn Software (ComboSyn Inc., Paramus, NJ, USA).

\section{D cultures from patient-derived ascitic cell}

For sphere forming capacity, primary cultured ascitic cells were reverse transfected with Lipofectamine $2000^{\circledR}$ and Negative Control or miR-654 mimic (25 nM) and seeded at $15 \times 10^{3}$ in non-adherent 24-well plates (coated with $0.5 \%$ agar in non-supplemented medium), in serum-free medium (1:1-MCDB105:M199, Biological Industries) supplemented with 1X B27 (Invitrogen), $2 \mathrm{mM}$ L-glutamine, and $20 \mathrm{ng} /$ $\mathrm{mL}$ EGF and FGF (ProSpec-Tany Technogene Ltd). At 
$96 \mathrm{~h}$, spheres were collected for protein extraction, MTS assay and for scoring. For MTS assays, tumor spheres were washed with PBS $1 \times$, and disaggregated with $0.5 \mathrm{~mL} 1 \mathrm{X}$ StemPro $^{\circledast}$ Accutase $^{\circledast}$ (ThermoFisher). PMS:MTS (1:20) mixture was added 1:10 to each well containing $100 \mu \mathrm{L}$ of disaggregated cells, and measured at $2-5 \mathrm{~h}$.

\section{Luciferase reporter assays}

DNA fragments containing the miR-654 binding sites for each 3'UTR region (and their corresponding 3'UTR mutated regions) of $H A X 1, C D C P 1, P B X 3, P L A G L 2$ and RAB1B genes were synthesized (GeneArt ${ }^{\mathrm{TM}}$ String ${ }^{\mathrm{TM}}$, ThermoFisher), amplified into the $\mathrm{pCR}^{\mathrm{TM}} \mathrm{II}-\mathrm{Blunt}-\mathrm{TOPO}^{\circledR}$ vector (Invitrogen), and cloned into the $\mathrm{psiCHECK}^{\mathrm{TM}}-2$ vector by using XhoI and NotI restriction sites (Supplementary Table 1k). 3'UTR luciferase reporter assays were performed as previously described [49]. Briefly, HEK293T cells were seeded at $2 \times 10^{4}$ cells/p96-well plates and co-transfected with $50 \mathrm{ng}$ of each $\mathrm{psiCHECK}^{\mathrm{TM}}-2-3^{\prime} \mathrm{UTR}$ vector and $25 \mathrm{nM}$ of miR-654 mimic or Negative Control. Luciferase assays were performed after $24 \mathrm{~h}$ using the Dual-Glo ${ }^{\circledR}$ Luciferase Assay System (Promega) and renilla was normalized to the firefly luciferase activity.

\section{Mouse xenograft}

All procedures were carried under the approval of the Institutional Animal Care at VHIR (protocol number 85/ 12). $2 \times 10^{6}$ cells of SKOV3 explanted cells transfected with Negative Control and miR-654 mimics $(25 \mathrm{nM})$ were injected in 8-week-old female NMRI-nude mice (Janvier, $n=12$ /group) in $300 \mu \mathrm{L}$ of PBS $1 \mathrm{X}$ and Matrigel (1:1). Tumor volume was measured every 2-3 days using an electronic caliper, and at day 25 mice were sacrificed and tumors were removed and weighed. Each tumor was used for $\mathrm{H} \& \mathrm{E}$ and Ki67 staining and for RNA extraction.

\section{Human mRNA microarray analyses}

SKOV3 cells transfected with siR-Control, siCDCP1 or siPLAGL2 $(n=3)$ were used in the microarray analysis. RNA concentration and quality were measured by Nanodrop and RNA Nano Chip Bioanalyzer (Agilent Technologies), and efficiency of CDCP1 and PLAGL2 depletion was measured by RTqPCR. Human Clariom ${ }^{\mathrm{TM}} \mathrm{S}$ assay (Affymetrix) was used to analyze whole-transcriptome expression profiling, which detects more than 20,000 well-annotated genes. The High Technology Unit (UAT) at VHIR performed all the steps after RNA extraction, including the first and second strand cDNA production, biotinylation, hybridization, labeling and scanning of the chips. Microarray raw data is publicly available at the GEOarchive repository GSE113115.

\section{Statistics}

Sample size was chosen to ensure adequate statistical power, according to the availability of the samples at the VHUH and the prevalence of the disease. Unless otherwise indicated, mean \pm SEM values are the average of three independent experiments. Statistical significance was determined by unpaired Student's $t$-test, assuming similar variances (GraphPad Prism Software, La Jolla, CA, USA). $* p<0.05 ; * * p<0.01 ; * * * p<0.001$ and $* * * * p<0.0001$. Identification of possible outliers was performed in all human data (ROUT method), the normal distribution of the data was verified (Shapiro-Wilk and Kolmogorov tests) and, accordingly, non-parametric Mann-Whitney test was used for comparisons between groups when analyzing human ovarian tissues. Compute correlations between pairs of data were performed by parametric Pearson and non-parametric Spearman correlation analyses.

Acknowledgements We are thankful to Drs. Diego Arango and Agueda Martinez, Dr. Sung Goo Park and Dr. Rosanna Paciucci for 9E10 c-Myc, anti-HAX1 and anti-RB and anti-p-RB antibodies, respectively. We thank Dr. Francesc Viñals, Dr. Barbara Vanderhyden, Dr. Antonio Rosato, Dr. Erich A. Nigg and the Ovarian Cancer Research Team for cell lines. We are grateful to Drs. Aroa Soriano and Luz Jubierre for experimental help. We acknowledge technical support from the Flow Cytometry Facility at Center of Genomic Regulation (CRG) and the Unitat d'Estadística i Bioinformàtica (UEB) and the Unitat d'Alta Tecnologia (UAT) at VHIR. We thank all our lab members for support and helpful discussions. This work was supported in part by grants from Instituto de la Mujer Dexeus (DEXEUSB29/012), CIBER (CB16/12/00328), SGR (2017 SGR 1661), the Ministerio de Economia y Competitividad and Fondos FEDER (RTC2015-3821-1), Instituto Carlos III (PI15/00238 to A.S. and PI17/00564 to M.F.S) and the Miguel Servet Program (CP13/00158 and CPII18/ 00027 to AS. and CPII16/00006 to MFS). AP and LS were supported by predoctoral VHIR fellowships and CJ by an AGAUR predoctoral fellowship (VHIR: PRED-VHIR-2014-11 and PRED-VHIR-2017; AGAUR: 2017FI_B_00095, respectively).

Author contributions BM and AS conceived and designed the study with help of AP and MFS. BM performed the miRNA and mRNA microarray analyses, and CJ carried out the mRNA microarray statistics and bioinformatics. BM and AP carried out all experiments and data analyses with the help of LS and MB. AM and GT provided technical support. ME and SM performed the DNA methylation analyses. JC analyzed and interpreted the IHC. JLS, APB, and AGM coordinated samples collection at HUVH and XMG, GM, FA, JP, and JAL provided with OC samples for the multicenter study. JR and MR initiated the miRNA screening. BM and AS wrote the manuscript with input from all other authors. AS supervised the project.

\section{Compliance with ethical standards}

Conflict of interest The authors declare that they have no conflict of interest.

Publisher's note: Springer Nature remains neutral with regard to jurisdictional claims in published maps and institutional affiliations. 


\section{References}

1. Siegel RL, Miller KD, Jemal A. Cancer statistics, 2017. CA. 2017;67:7-30.

2. Karnezis AN, Cho KR, Gilks CB, Pearce CL, Huntsman DG. The disparate origins of ovarian cancers: pathogenesis and prevention strategies. Nat Rev Cancer. 2016;17:65-74.

3. Matulonis UA, Sood AK, Fallowfield L, Howitt BE, Sehouli J, Karlan BY. Ovarian cancer. Nat Rev Dis Prim. 2016;2:16061.

4. Ueland F. A perspective on ovarian cancer biomarkers: past, present and yet-to-come. Diagnostics. 2017;7:14.

5. Kipps E, Tan DSP, Kaye SB. Meeting the challenge of ascites in ovarian cancer: new avenues for therapy and research. Nat Rev Cancer. 2013;13:273-82.

6. Vaughan S, Coward JI, Bast RC, Berchuck A, Berek JS, Brenton $\mathrm{JD}$, et al. Rethinking ovarian cancer: recommendations for improving outcomes. Nat Rev Cancer. 2011;11:719-25.

7. Banerjee S, Kaye SB. New strategies in the treatment of ovarian cancer: current clinical perspectives and future potential. Clin Cancer Res. 2013;19:961-8.

8. Sehouli J, Braicu E, Chekerov R. PARP inhibitors for recurrent ovarian carcinoma: current treatment options and future perspectives. Geburtshilfe Frau. 2016;76:164-9.

9. George A, Kaye S, Banerjee S. Delivering widespread BRCA testing and PARP inhibition to patients with ovarian cancer. Nat Rev Clin Oncol. 2016. https://doi.org/10.1038/nrclinonc.2016.191.

10. Rupaimoole R, Slack FJ. MicroRNA therapeutics: towards a new era for the management of cancer and other diseases. Nat Rev Drug Disco. 2017;16:203-22.

11. Ji W, Sun B, Su C. Targeting microRNAs in cancer gene therapy. Genes. 2017;8:21.

12. Prahm KP, Novotny GW, Høgdall C, Høgdall E. Current status on microRNAs as biomarkers for ovarian cancer. APMIS. 2016;124:337-55.

13. Zhang L, Volinia S, Bonome T, Calin GA, Greshock J, Yang N, et al. Genomic and epigenetic alterations deregulate microRNA expression in human epithelial ovarian cancer. Proc Natl Acad Sci. 2008;105:7004-9.

14. Yang D, Sun Y, Hu L, Zheng H, Ji P, Pecot CV, et al. Integrated analyses identify a master microRNA regulatory network for the mesenchymal subtype in serous ovarian cancer. Cancer Cell. 2013;23:186-99.

15. Mateescu B, Batista L, Cardon M, Gruosso T, de Feraudy Y, Mariani O, et al. MiR-141 and miR-200a act on ovarian tumorigenesis by controlling oxidative stress response. Nat Med. 2011;17:1627-35.

16. Bell D, Berchuck A, Birrer M, Chien J, Cramer DW, Dao F, et al. Integrated genomic analyses of ovarian carcinoma. Nature. 2011;474:609-15.

17. Beg MS, Brenner AJ, Sachdev J, Borad M, Kang Y-K, Stoudemire J, et al. Phase I study of MRX34, a liposomal miR-34a mimic, administered twice weekly in patients with advanced solid tumors. Invest New Drugs. 2017;35:180-8.

18. Zehavi L, Avraham R, Barzilai A, Bar-Ilan D, Navon R, Sidi Y, et al. Silencing of a large microRNA cluster on human chromosome $14 \mathrm{q} 32$ in melanoma: biological effects of mir-376a and mir$376 \mathrm{c}$ on insulin growth factor 1 receptor. Mol Cancer. 2012;11:44.

19. Maire G, Martin JW, Yoshimoto M, Chilton-MacNeill S, Zielenska M, Squire JA. Analysis of miRNA-gene expressiongenomic profiles reveals complex mechanisms of microRNA deregulation in osteosarcoma. Cancer Genet. 2011;204:138-46.

20. Takahashi M, Tsukamoto Y, Kai T, Tokunaga A, Nakada C, Hijiya N, et al. Downregulation of WDR20 due to loss of $14 \mathrm{q}$ is involved in the malignant transformation of clear cell renal cell carcinoma. Cancer Sci. 2016;107:417-23.
21. Li J, Zhou D, Wang Z, Tan L, Zhou Y, Li J, et al. Reversal effect of 5-aza-2-deoxycytidine on the maternally expressed gene 3 promoter hypermethylation and its inhibitory effect on the proliferation of epithelial ovarian cancer cells. Zhonghua Zhong Liu Za Zhi. 2015;37:324-9.

22. Adair SJ, Hogan KT. Treatment of ovarian cancer cell lines with 5-aza-2'-deoxycytidine upregulates the expression of cancer-testis antigens and class I major histocompatibility complex-encoded molecules. Cancer Immunol Immunother. 2009;58:589-601.

23. Jacob F, Hitchins MP, Fedier A, Brennan K, Nixdorf S, Hacker $\mathrm{NF}$, et al. Expression of GBGT1 is epigenetically regulated by DNA methylation in ovarian cancer cells. BMC Mol Biol. 2014;15:24.

24. Chen M-W, Yang S-T, Chien M-H, Hua K-T, Wu C-J, Hsiao SM, et al. The STAT3-miRNA-92-Wnt signaling pathway regulates spheroid formation and malignant progression in ovarian cancer. Cancer Res. 2017;77:1955-67.

25. Honkoop AH, Pinedo HM, De Jong JS, Verheul HM, Linn SC, Hoekman K, et al. Effects of chemotherapy on pathologic and biologic characteristics of locally advanced breast cancer. Am J Clin Pathol. 1997;107:211-8.

26. Dweep H, Gretz N. MiRWalk2.0: a comprehensive atlas of microRNA-target interactions. Nat Methods. 2015;12:697.

27. Sato E, Olson SH, Ahn J, Bundy B, Nishikawa H, Qian F, et al. Intraepithelial CD8 + tumor-infiltrating lymphocytes and a high $\mathrm{CD} 8+$ /regulatory $\mathrm{T}$ cell ratio are associated with favorable prognosis in ovarian cancer. Proc Natl Acad Sci. 2005;102:18538-43.

28. Bi L, Yang Q, Yuan J, Miao Q, Duan L, Li F, et al. MicroRNA127-3p acts as a tumor suppressor in epithelial ovarian cancer by regulating the BAG5 gene. Oncol Rep. 2016. https://doi.org/10. 3892/or.2016.5055.

29. Shepherd TG, Thériault BL, Campbell EJ, Nachtigal MW. Primary culture of ovarian surface epithelial cells and ascites-derived ovarian cancer cells from patients. Nat Protoc. 2007;1:2643-9.

30. Cummins JM, He Y, Leary RJ, Pagliarini R, Diaz LA, Sjoblom T, et al. The colorectal microRNAome. Proc Natl Acad Sci USA. 2006;103:3687-92.

31. Paydas S, Acikalin A, Ergin M, Celik H, Yavuz B, Tanriverdi K. Micro-RNA (miRNA) profile in Hodgkin lymphoma: association between clinical and pathological variables. Med Oncol. 2016;33:34.

32. Tan Y-Y, Xu X-Y, Wang J-F, Zhang C-W, Zhang S-C. MiR-654$5 p$ attenuates breast cancer progression by targeting EPSTI1. Am J Cancer Res. 2016;6:522-32.

33. Kircher M, Bock C, Paulsen M. Structural conservation versus functional divergence of maternally expressed microRNAs in the Dlk1/Gt12 imprinting region. BMC Genom. 2008;9:346.

34. Ostling $\mathrm{P}$, Leivonen S-K, Aakula A, Kohonen P, Makela R, Hagman Z, et al. Systematic analysis of microRNAs targeting the androgen receptor in prostate cancer cells. Cancer Res. 2011;71:1956-67.

35. Landrette SF, Kuo YH, Hensen K, Barjesteh van Waalwijk van Doorn-Khosrovani S, Perrat PN, Van de Ven WJ, et al. Plag1 and Plag12 are oncogenes that induce acute myeloid leukemia in cooperation with Cbfb-MYH11. Blood. 2005;105:2900-7.

36. He Y, Wu AC, Harrington BS, Davies CM, Wallace SJ, Adams $\mathrm{MN}$, et al. Elevated CDCP1 predicts poor patient outcome and mediates ovarian clear cell carcinoma by promoting tumor spheroid formation, cell migration and chemoresistance. Oncogene. 2016;35:468-78.

37. Harrington BS, He Y, Davies CM, Wallace SJ, Adams MN, Beaven EA, et al. Cell line and patient-derived xenograft models reveal elevated CDCP1 as a target in high-grade serous ovarian cancer. Br J Cancer. 2016;114:417-26.

38. Hensen K, Van Valckenborgh ICC, Kas K, Van de Ven WJM, Voz ML. The tumorigenic diversity of the three PLAG family 
members is associated with different DNA binding capacities. Cancer Res. 2002;62:1510-7.

39. Landrette SF, Madera D, He F, Castilla LH. The transcription factor PlagL2 activates Mpl transcription and signaling in hematopoietic progenitor and leukemia cells. Leukemia. 2011;25:655-62.

40. Poole CJ, van Riggelen J. MYC-Master regulator of the cancer epigenome and transcriptome. Genes. 2017. https://doi.org/10. 3390/genes8050142.

41. Reyes-Gonzalez JM, Armaiz-Pena GN, Mangala LS, Valiyeva F, Ivan C, Pradeep S, et al. Targeting c-MYC in platinum-resistant ovarian cancer. Mol Cancer Ther. 2015;14:2260-9.

42. Zheng H, Ying H, Wiedemeyer R, Yan H, Quayle SN, Ivanova $\mathrm{EV}$, et al. PLAGL2 regulates Wnt signaling to impede differentiation in neural stem cells and gliomas. Cancer Cell. 2010;17:497-509.

43. Wang Y-P, Guo P-T, Zhu Z, Zhang H, Xu Y, Chen Y-Z et al. Pleomorphic adenoma gene like-2 induces epithelialmesenchymal transition via $\mathrm{Wnt} / \mathrm{b}$-catenin signaling pathway in human colorectal adenocarcinoma. Oncol Rep. 2017. https://doi. org/10.3892/or.2017.5485.
44. Livak KJST. Analysis of relative gene expression data using realtime quantitative PCR and the 2(-Delta Delta $\mathrm{C}(\mathrm{T})$ ) method. Methods. 2001;25:402-8.

45. Klinkebiel D, Zhang W, Akers SN, Odunsi K, Karpf AR. DNA methylome analyses implicate fallopian tube epithelia as the origin for high-grade serous ovarian cancer. Mol Cancer Res. 2016;14:787-94.

46. Iorio F, Knijnenburg TA, Vis DJ, Bignell GR, Menden MP, Schubert $\mathrm{M}$, et al. A landscape of pharmacogenomic interactions in cancer. Cell. 2016;166:740-54.

47. Moran S, Vizoso M, Martinez-Cardus A, Gomez A, Matias-Guiu $\mathrm{X}$, Chiavenna SM, et al. Validation of DNA methylation profiling in formalin-fixed paraffin-embedded samples using the infinium humanmethylation450 microarray. Epigenetics. 2014;9:829-33.

48. Jubierre L, Soriano A, Planells-Ferrer L, París-Coderch L, Tenbaum SP, Romero OA, et al. BRG1/SMARCA4 is essential for neuroblastoma cell viability through modulation of cell death and survival pathways. Oncogene. 2016;35:5179-90.

49. Soriano A, París-Coderch L, Jubierre L, Martínez A, Zhou X, Piskareva O, et al. MicroRNA-497 impairs the growth of chemoresistant neuroblastoma cells by targeting cell cycle, survival and vascular permeability genes. Oncotarget. 2016;7:9271-87.

\section{Affiliations}

\section{Blanca Majem ${ }^{1,18,18}$ - Alfonso Parrilla ${ }^{1} \cdot$ Carlos Jiménez $^{2} \cdot$ Leticia Suárez-Cabrera $^{1}$ - Marta Barber ${ }^{1}$ - Andrea Marín ${ }^{1}$. Josep Castellví ${ }^{3}$ - Gabriel Tamayo ${ }^{1}$ - Gema Moreno-Bueno ${ }^{4,5}$ - Jordi Ponce ${ }^{6}$ - Xavier Matias-Guiu, ${ }^{7,8}$. Francesc Alameda ${ }^{9}$ Ignacio Romero ${ }^{10}$ - José Luis Sánchez ${ }^{11,12}$. Asunción Pérez-Benavente ${ }^{11,12}$. Sebastián Moran $\mathbb{1}^{13} \cdot$ Manel Esteller ${ }^{13,14,15} \cdot$ Jaume Reventós ${ }^{8,16} \cdot$ Marina Rigau ${ }^{17}$. Antonio Gil-Moreno $\mathbb{D}^{11,12}$. Miguel F. Segura $\mathbb{D}^{2} \cdot$ Anna Santamaría ${ }^{1}$}

1 Cell Cycle and Cancer Laboratory, Group of Biomedical Research in Urology, Vall Hebron Research Institute (VHIR), Universitat Autònoma de Barcelona (UAB), 08035 Barcelona, Spain

2 Group of Translational Research in Child and Adolescent Cancer, Vall Hebron Research Institute (VHIR), Universitat Autònoma de Barcelona (UAB), 08035 Barcelona, Spain

3 Department of Pathology, Vall Hebron University Hospital, 08035 Barcelona, Spain

4 Department of Biochemistry, Universidad Autónoma de Madrid (UAM), Instituto de Investigaciones Biomédicas 'Alberto Sols' (CSIC-UAM), CIBERONC, IdiPaz, 28029 Madrid, Spain

5 Fundación MD Anderson Internacional, 28033 Madrid, Spain

6 Departement of Gynaecology, University Hospital of Bellvitge (IDIBELL), University of Barcelona, Barcelona 08908, Spain

7 Department of Pathology Hospital Universitari Arnau de Vilanova, University of Lleida, IRB-Lleida, Lleida 25198, Spain

8 Department of Pathology Hospital Universitari de Bellvitge, IDIBELL, CIBERONC, 08908 Barcelona, Spain

9 Pathology Department, Hospital del Mar, 08003 Barcelona, Spain

10 Department of Medical Oncology, Instituto Valenciano de Oncología (IVO), 46009 Valencia, Spain
11 Department of Gynecological Oncology, Vall Hebron University Hospital, Universitat Autònoma de Barcelona (UAB), 08035 Barcelona, Spain

12 Group of Biomedical Research in Gynecology, Vall Hebron Research Institute (VHIR), Universitat Autònoma de Barcelona (UAB), CIBERONC, 08035 Barcelona, Spain

13 Cancer Epigenetics and Biology Program (PEBC), Bellvitge Biomedical Research Institute (IDIBELL), CIBERONC, 08908 Barcelona, Spain

14 Physiological Sciences Department, School of Medicine and Health Sciences, University of Barcelona (UB), 08036 Barcelona, Spain

15 Institució Catalana de Recerca i Estudis Avançats (ICREA), 08010 Barcelona, Spain

16 Department of Basic Sciences, Universitat Internacional de Catalunya, 08017 Barcelona, Spain

17 Bellvitge Biomedical Research Institute (IDIBELL), 08908 Barcelona, Spain

18 Present address: Cell Death Regulation Group, Oncobell Program, Bellvitge Biomedical Research Institute (IDIBELL), L'Hospitalet de Llobregat, Barcelona 08908, Spain 